OPEN ACCESS

Edited by:

Julie Hardouin,

Université de Rouen, France

Reviewed by:

Feng Ge,

Institute of Hydrobiology, Chinese Academy of Sciences (CAS), China

Rahul Modak,

KIIT University, India

*Correspondence:

Julia Gallego-Jara

julia.gallego@um.es

Specialty section:

This article was submitted to

Microbial Physiology and Metabolism,

a section of the journal

Frontiers in Microbiology

Received: 20 July 2021 Accepted: 04 October 2021

Published: 26 October 2021

Citation:

Gallego-Jara J, Ortega Á, Lozano Terol G, Sola Martínez RA,

Cánovas Díaz M and

de Diego Puente T (2021) Bacterial

Sirtuins Overview: An Open Niche

to Explore.

Front. Microbiol. 12:744416. doi: 10.3389/fmicb.2021.744416

\section{Bacterial Sirtuins Overview: An Open Niche to Explore}

\author{
Julia Gallego-Jara*, Álvaro Ortega, Gema Lozano Terol, Rosa A. Sola Martínez, \\ Manuel Cánovas Díaz and Teresa de Diego Puente
}

Department of Biochemistry and Molecular Biology (B) and Immunology, Faculty of Chemistry, University of Murcia, Campus de Espinardo, Regional Campus of International Excellence "Campus Mare Nostrum", Murcia, Spain

Sirtuins are deacetylase enzymes widely distributed in all domains of life. Although for decades they have been related only to histones deacetylation in eukaryotic organisms, today they are considered global regulators in both prokaryotes and eukaryotes. Despite the important role of sirtuins in humans, the knowledge about bacterial sirtuins is still limited. Several proteomics studies have shown that bacterial sirtuins deacetylate a large number of lysines in vivo, although the effect that this deacetylation causes in most of them remains unknown. To date, only the regulation of a few bacterial sirtuin substrates has been characterized, being their metabolic roles widely distributed: carbon and nitrogen metabolism, DNA transcription, protein translation, or virulence. One of the most current topics on acetylation and deacetylation focuses on studying stoichiometry using quantitative LC-MS/MS. The results suggest that prokaryotic sirtuins deacetylate at low stoichiometry sites, although more studies are needed to know if it is a common characteristic of bacterial sirtuins and its biological significance. Unlike eukaryotic organisms, bacteria usually have one or few sirtuins, which have been reported to have closer phylogenetic similarity with the human Sirt5 than with any other human sirtuin. In this work, in addition to carrying out an in-depth review of the role of bacterial sirtuins in their physiology, a phylogenetic study has been performed that reveals the evolutionary differences between sirtuins of different bacterial species and even between homologous sirtuins.

Keywords: sirtuins, bacteria, deacetylation, metabolism, prokaryote

\section{LYSINE ACETYLATION IS A GLOBAL POST-TRANSLATIONAL MODIFICATION}

Protein acetylation is a post-translational modification (PTM) consisting of the transfer of an acetyl group from an acetyl donor to a protein residue. Two different types of protein acetylation have been identified, $N$ - $\alpha$-acetylation or terminal acetylation and $N$ - $\varepsilon$-acetylation or lysine acetylation (Drazic et al., 2016). In $N$ - $\alpha$-acetylation, the transfer of an acetyl group from acetyl-CoA to an $\mathrm{N}$-terminal residue of a peptide or protein is catalyzed by $\mathrm{N}$-acetyltransferases (NATs) (Drazic et al., 2016). N-terminal acetylation is frequent in eukaryotic organisms, although it is proposed to be less common in bacteria (Nguyen et al., 2018; Parks and Escalante-Semerena, 2020). In contrast, $N$ - $\varepsilon$-acetylation is a PTM widely distributed in all domains of life (Wagner and Payne, 2013; Macek et al., 2019). $N$ - $\varepsilon$-acetylation consists of the transfer of an acetyl group from acetyl-CoA 
or acetyl-phosphate to the $N-\varepsilon$ group of a lysine residue of a protein. Moreover, unlike $\mathrm{N}$-terminal acetylation, $\mathrm{N}-\varepsilon$ acetylation can occur without the participation of any enzyme (Wagner and Payne, 2013; Kuhn et al., 2014; James et al., 2017). $N$ - $\varepsilon$-acetylation was for decades only related to histone acetylation by histone acetyltransferases (HATs) (Hebbes et al., 1988). However, advances in mass spectrometry allowed the identification of many non-histone acetylated proteins in the three domains of life (Spange et al., 2009; Zhang et al., 2009). Due to the wide diversity of acetylated proteins, histone acetyltransferases were renamed as lysine acetyltransferases (KATs). Regarding $N$ - $\varepsilon$-acetylation in bacteria, the first bacterial lysine acetylome was reported in E. coli in 2008. Subsequently, several acetylome studies have been reported across bacterial species (Christensen et al., 2019). These studies have shown that protein acetylation is one of the most frequent PTM, highly conserved and ancient in prokaryotes (Yu et al., 2008; Crosby et al., 2012; Wua et al., 2013; Castaño-Cerezo et al., 2014; Kosono et al., 2015; Ouidir et al., 2015; Xie et al., 2015; Nakayasu et al., 2017; Wei et al., 2017; Li et al., 2018).

Bacterial acetylation has become an important mechanism to regulate metabolism and virulence allowing organisms their adaptation to different environments (Bernal et al., 2014; Nurulain and Zaveri, 2016; Carabetta and Cristea, 2017; Macek et al., 2019). Acetylation can occur by two different mechanisms, catalyzed by KATs or through a non-enzymatic mechanism. Nonenzymatic lysine acetylation is positioned as the most frequent mechanism of acetylation in the prokaryotic model Escherichia coli (E. coli). Furthermore, lysines that are acetylated by a KAT are not usually non-enzymatically acetylated, and vice versa (Weinert et al., 2013a; Kuhn et al., 2014; Christensen et al., 2018). The importance of lysine acetylation in metabolism regulation depends, in part, on its reversibility. Acetylation of proteins can be reverted by lysine deacetylases (KDACs). Depending on their sequence and domain organization, KDACs have been divided into four groups: class I, II, and IV KDACs need $\mathrm{Zn}^{2+}$, while class III, also known as Sir-2 like proteins or sirtuins, need oxidized nicotinamide adenine dinucleotide $\left(\mathrm{NAD}^{+}\right)$to carry out protein deacetylation (Frye, 2000; Gregoretti et al., 2004). Interestingly, a proteomic study carried out by AbouElfetouh et al. (2015) demonstrated that in E. coli, CobB sirtuin can deacetylate lysines both enzymatically and non-enzymatically acetylated, without a clear preference. Furthermore, this study identified 69 lysines reproducibly, significantly, and robustly more acetylated in a deficient $c o b B$ strain than in wild type. Nevertheless, most nonenzymatic acetylation events do not appear to be reversed by a KDAC (Weinert et al., 2013a; Kuhn et al., 2014; AbouElfetouh et al., 2015).

Beyond the identification of acetylation and deacetylation points in proteins and acetylation fold-changes under different conditions or mutants, precise measures of acetylation site occupancy, or stoichiometry, were not possible until a few years ago. In recent years the development of quantitative LCMS/MS methods has allowed the study of the stoichiometry of acetylation and deacetylation (Baeza et al., 2014; Meyer et al., 2016; Gil et al., 2017; Weinert et al., 2017). Acetylation or deacetylation stoichiometry of a modificated lysine refers to the fraction of that lysine that is acetylated or deacetylated. To our knowledge, two works have studied how the deletion of $E$. coli $\operatorname{cobB}$ gene affects the acetylation stoichiometry, concluding that the absence of CobB increases acetylation at low stoichiometry sites, which had also been demonstrated for human Sirt3 (Baeza et al., 2014; Weinert et al., 2015, 2017). This result and the similarities between CobB and SIRT3 suggest an evolutionary origin of sirtuin deacetylases and a promiscuous activity, which needs to be further investigated to know if it is a common characteristic of all sirtuins. The fact that deacetylation occurs at very low stoichiometries could indicate a low impact of this modification on overall activity, however, it is not necessarily true, because deacetylation may be carried out on a small fraction of proteins that are spatially or temporally different from the rest (Olsen and Mann, 2013). The stoichiometry of sirtuin-mediated deacetylation is essential to know its real physiological role, and how it changes according to environmental conditions. Despite the advances made in recent years in this field, it is necessary to continue the study of the stoichiometry of acetylation and deacetylation in other organisms, both prokaryotic and eukaryotic.

\section{SIRTUINS: A REGULATORY FAMILY OF PROTEINS CONSERVED SINCE BACTERIA TO HUMANS}

Sirtuins are lysine deacylase enzymes consuming $\mathrm{NAD}^{+}$and producing nicotinamide (NAM) and 2'-O-acetyl-ADP-ribose. In the 1970s, the first sirtuin was identified in Saccharomyces cerevisiae (S. cerevisiae) and was named Sir2 (Silent informator regulator 2) (Klar et al., 1979; Ivy et al., 1986). However, its essential role on replicative lifespan and deacetylase activity was not discovered until much later (Kennedy et al., 1995; Kaeberlein et al., 1999; Imai et al., 2000). Since the discovery of Sir2, several Sir2-like proteins were identified in prokaryotes and eukaryotes organisms, and Sir2-like proteins became known as sirtuins (Brachmann et al., 1995). The role of sirtuins in eukaryotic organisms has been widely studied and, to date, seven Sir2 like deacetylases (Sirt1-Sirt7) have been identified in human cells.

Sirtuins share an approximately 275 amino acid long conserved core and a variable $\mathrm{N}$ - and C-terminal domains (Costantini et al., 2013). In the conserved core exists a $\mathrm{NAD}^{+}$ binding domain consisting in a Rossmann fold and a $\mathrm{Zn}^{2+}$ binding domain coordinated with four Cys residues in a tetrahedral geometry. These two domains are linked by a loop area where the acetylated peptide is accommodated. Regarding the area of substrate binding to the catalytic domain, sirtuins show three pockets: (A) where the ADP-ribose intermediate binds, (B) where the nicotinamide-ribose interaction occurs, and (C) where the nicotinamide is located, allowing the transfer of the acetyl group from lysine to ribose (Avalos et al., 2002; Yuan and Marmorstein, 2012). Beyond the central core conserved in all sirtuins, the length and structure of the terminal amino and carboxyl are highly variable, with several intrinsically disordered structures that are proposed to be involved in the 
determination of the specificity and subcellular location of the sirtuin (Costantini et al., 2013).

The first bacterial sirtuin was identified in Salmonella enterica (S. enterica) and was called CobB because it was related to cobalamin biosynthesis and propionic catabolism (Tsang and Escalante-Semerena, 1996; Tsang and Escalante-semerena, 1998). Shortly after, deacetylation of acetyl-CoA synthetase (Acs) in S. enterica by CobB was characterized (Starai et al., 2002). This discovery meant a turning point in sirtuin research: the regulation by $\mathrm{N}-\varepsilon$ deacetylation of a non-histone protein was characterized for the first time. Shortly after this first identification in Salmonella, a bacterial sirtuin, CobB from E. coli, was crystallized and its structure solved, concluding that cognate substrate binding involves the $\mathrm{Zn}$-binding domain and regions distal to the acetyl-lysine pocket (Zhao et al., 2004).

Escherichia coli CobB was identified as the first prokaryotic desuccinylase enzyme (Colak et al., 2013). Succinylation, previously identified in mitochondria, has been described as a frequent modification in prokaryotes, overlapping extensively with acetylation (Du et al., 2011; Weinert et al., 2013b). In Bacillus subtilis (B. subtilis), proteomic studies have identified hundreds of acetylated and succinylated proteins. The increase in the acetylation and succinylation observed in the two B. subtilis deacetylase deficient strains, $\Delta a c u C \Delta s r t N$, suggests a role of both of them in the global deacetylation and desuccinylation systems (Kim et al., 2013; Kosono et al., 2015). In this sense, a recent study carried out a kinetic characterization of SrtN over different acylated substrates concluding that this sirtuin catalyzes efficiently other deacylations different to acetylation, such as malonylation or formylation (Seidel et al., 2016). Propionylation has also been reported to play a vital role in cellular physiology of prokaryotes and eukaryotes. It is a reversible PTM, depropionylation being catalyzed by sirtuins as human Sirt1 or CobB from E. coli and S. enterica (Garrity et al., 2007; Cheng et al., 2009; Sun et al., 2016b). In addition, some sirtuins, like the Lactobacillus acidophilus (L. acidophilus) Sir2La, the unique characterized sirtuin from L. acidophilus, shows a preference for propionylated over acetylated and butyrylated substrates (Olesen et al., 2018).

Beyond "classical" deacylations, sirtuins are related to other deacylation reactions such as delipoamidation or dehomocysteinylation. Lipoamidation is a modification identified in eukaryotes in the 1950s responsible for the regulation of multimeric enzyme complexes. However, it has been poorly studied in prokaryotes (Reed et al., 1958; Reed, 2001). Reversible lipoamidation has been identified in eukaryotes and prokaryotes, being $\mathrm{CobB}$ the first bacterial sirtuin with delipoamidase activity (Mathias et al., 2014; Rowland et al., 2017). In E. coli, CobB regulates pyruvate dehydrogenase $(\mathrm{PDH})$ and ketoglutarate dehydrogenase $(\mathrm{KDH})$ complexes, two carbon entry points into the tricarboxylic acid cycle, by delipoamidation, which highlights the role in central metabolism of this sirtuin. Lysine dehomocysteinylation activity has been attributed to $S$. enterica CobB, the only prokaryotic enzyme with this activity to date. This interesting finding suggests that sirtuins might play a role in hyperhomocysteinemia, the increase of homocysteinylated proteins in human which is related to many diseases (Mei et al., 2016). In addition to these deacylase activities, some sirtuins also catalyze ADP ribosylations, a modification consisting in the transfer of the ADP-ribose group from $\mathrm{NAD}^{+}$to an acceptor, producing mono-ADP-ribosylated proteins and nicotinamide (Appel et al., 2016). In bacteria, auto ADP-ribosylation of a sirtuin from Mycobacterium smegmatis (M. smegmatis) has been identified as essential for growth in natural environments (Tan et al., 2015). ADP-ribosylation activity has been also associated to sirtuins from microbial pathogens (Rack et al., 2015).

\section{SIRTUINS IN CARBON AND NITROGEN METABOLISM}

Deacetylation of acetylated Acs K609 by CobB in S. enterica (also known SeCobB) was the first described bacterial sirtuin reaction (Starai et al., 2002, 2003). Following this milestone, Acs protein has been shown to be a substrate for other sirtuins in many prokaryotic and eukaryotic organisms (Schwer et al., 2006; Gardner and Escalante-Semerena, 2009; Crosby et al., 2010; Xu et al., 2011; Mikulik et al., 2012; Hayden et al., 2013; Tucker and Escalante-Semerena, 2013; De Diego Puente et al., 2015; VanDrisse and Escalante-Semerena, 2018; Burckhardt et al., 2019). A recent study has shown that the binding of cAMP to Acs inhibits its deacetylation by SeCobB (Han et al., 2017). Our group carried out the kinetic characterization of $E$. coli Acs deacetylation by CobB, showing that deacetylated a minimum of 20 acetylated lysines of Acs with a single kinetic rate, which suggests that the main determinants of sirtuin specificity are the structural protein components rather than the protein sequence. This is, to our knowledge, the only sirtuin kinetic characterization with a fully acetylated and natively folded protein as a substrate (Gallego-Jara et al., 2017). Acs from B. subtilis, AcsA, is essential to consume the acetate present in soil, the natural habitat of this bacterium (Grundy et al., 1993). The activity of B. subtilis AcsA has been reported to be regulated by acetylation/deacetylation. However, while acetylation is catalyzed by AcuA acetyltransferase, two lysine deacetylases are involved in its deacetylation: $\mathrm{NAD}^{+}$ independent AcuC and sirtuin SrtN (Gardner et al., 2006; Gardner and Escalante-Semerena, 2009).

Rhodopseudomonas palustris ( $R$. palustris) is a photoheterotrophic bacterium able to degrade aromatic compounds to acetyl-CoA, which makes it an interesting weapon against environmental contamination by humanproduced aromatic compounds. In $R$. palustris, reversible acetylation regulates acyl-CoA synthetases responsible for aromatic activation. Interestingly, activation of three of these acyl-CoA synthetases (Benzoyl-CoA (BadA), 4-hydroxybenzoylCoA (HbaA) and cyclohexanecarboxyl-CoA (AliA) synthases) through lysine deacetylation can be catalyzed by the sirtuin RpSrtN or by the Zn-dependent protein deacetylase LdaA (Crosby et al., 2010, 2012).

In 2007, Garrity et al. demonstrated that expression of CobB in S. enterica was necessary for it to grow with acetate or propionate as the only carbon source. The CobB activity need for growth in propionate triggered the identification of the enzyme 
PrpE (propionyl-CoA synthetase) as a new substrate of this sirtuin. In this case, SeCobB catalyzed the depropionylation of the 592 PrpE lysine, which induced its activation. This study was the first to reveal an activity different to deacetylation in a bacterial sirtuin (Garrity et al., 2007). In Mycobacterium tuberculosis and $M$. smegmatis reversible acetylation is also involved in propionate metabolism through acetylation and deacetylation carried out by the Rv1151c sirtuin or the ortholog in M. smegmatis, SrtN (Hayden et al., 2013; Nambi et al., 2013). Interestingly, while Rv1151c is the only found Sir2 like protein in M. tuberculosis, two predicted sirtuins, Ms5175 and Ms4620, are observed in M. smegmatis, showing Ms4620 a robust ADPribosylation activity (Tan et al., 2015). M. tuberculosis fatty acyl CoA synthetases (FadD22, FadD2, FadD5, and FadD13), AMPforming acyl-CoA synthetases similar to Acs, are also acetylated and deacetylated by Rv1151. This modification activates fatty acids metabolism (Nambi et al., 2013). Further, FadD33, a M. smegmatis fatty acid synthetase that is indispensable for Mycobacterium virulence development through siderophores, is also regulated by acetylation and deacetylation through its $\mathrm{SrtN}$ sirtuin (Vergnolle et al., 2013).

Beyond acyl-CoA synthetases, other metabolic enzymes have been reported to be regulated by acetylation/deacetylation. For example, the $S$. enterica's glyceraldehyde-3-phosphate dehydrogenase (GapA), isocitrate lyase (Icl), or isocitrate dehydrogenase phosphatase/kinase (AceK) enzymes (Wang et al., 2010). Results from this study were questioned however in other reports (Crosby et al., 2012; Crosby and EscalanteSemerena, 2014). Isocitrate lyase from $M$. tuberculosis is also regulated by sirtuin deacetylation (Bi et al., 2017). The first substrate of CobB identified in E. coli was the chemotactic regulator CheY (Li et al., 2010). CheY is an essential regulator of chemotaxis, the mechanism through which bacteria respond to changes in the chemical compositions of their environment. In addition to $\mathrm{CheY}$, the $\mathrm{N}$-hydroxyarylamine $\mathrm{O}$-acetyltransferase (NhoA) protein, the citrate synthase (Cs) and the isocitrate dehydrogenase ( $\mathrm{IcdH}$ ) enzymes, both of them belonging to the tricarboxylic acids cycle, and the adenosylmethionine synthase (MetK) are also substrates of CobB sirtuin (Zhang et al., 2013; Sun et al., 2016a; Venkat et al., 2018, 2019). Recently, the activity and stability of $E$. coli diguanylate cyclase $\mathrm{DgcZ}$ has also been demonstrated to be regulated by deacetylation catalyzed by CobB sirtuin (Xu et al., 2019). Furthermore, CobB regulates TCA cycle by delipoamidation $\mathrm{PDH}$ and $\mathrm{KDH}$ complexes (Rowland et al., 2017).

In 2016, nitrogen metabolism of Saccharopolyspora erythraea (S. erythraea) was shown to be regulated by sirtuin-dependent reversible lysine acetylation of two glutamine synthetases, GlnA1 and $\mathrm{G} \ln \mathrm{A} 4$. While acetylation inactivated $\mathrm{G} \ln \mathrm{A} 4$, acetylation of GlnA1 led to a fine modulation of the nitrogen regulator GlnR binding to DNA with a chaperone-like activity. Moreover, GlnR regulates transcription of AcuA acetyltransferase and of SacSrtN sirtuin, responsible for GlnA1 and GlnA4 acetylation and deacetylation, respectively, forming an autofeedback loop in nitrogen metabolism. This function of the acetylated GlnA1 over GlnR regulator seems to be conserved through actinomycetes such as S. coelicolor and M. smegmatis (You et al., 2016). GlnR also promotes transcription of the three AMP forming acetylCoA synthetases from $S$. erythraea: acs $A 1$, acs $A 2$, and acs $A 3$ in response to nitrogen availability. Their activity is regulated by lysine acetylation catalyzed by the acetyltransferase AcuA and the sirtuin SacSrtN (You et al., 2014, 2017). A more recent study also highlights the role of the SacSrtN sirtuin beyond $\mathrm{G} \ln \mathrm{A} 1$ and $\mathrm{G} \ln \mathrm{A} 4$ deacetylation. It suggests a central role of SacSrtN in the regulation of the primary and secondary metabolism in S. erythraea (Zhou et al., 2018). Role of bacterial sirtuins in carbon and nitrogen metabolism is resumed in Figure 1.

\section{SIRTUINS IN BACTERIAL VIRULENCE AND RESISTANCE TO STRESS}

Salmonella enterica is a facultative pathogen that survives within the cells of its host. TacT is a toxin acetyltransferase identified in S. enterica and responsible for antitoxin TacA acetylation, which is deacetylated by $\mathrm{SeCobB}$ sirtuin (VanDrisse et al., 2017). TacA acetylation increases TacT activity and therefore the persistence state inside a host. The two-component system PhoP-PhoQ is also involved in S. enterica virulence. Its activity is regulated by acetylation at K201 reverted by sirtuin SeCobB (Ren et al., 2016). Results showed that deacetylation of PhoP at K201 is essential for Salmonella pathogenesis. These data support the role of acetylation/deacetylation by SeCobB as a key regulator in S. enterica virulence (Yu et al., 2016). Reversible acetylation has also been related to $S$. enterica and $E$. coli survival under acid stress through modulation of Pat and CobB activity (Ma and Wood, 2011; Ren et al., 2015).

Role of sirtuin in Yersinia pestis ( $Y$. pestis), responsible for causing pulmonary, bubonic and also septicemic plague in humans, has also been studied. Thus, Y. pestis reversible acetylation catalyzed by Pat acetyltransferase and CobB-like sirtuin, $\mathrm{YpCobB}$, regulates the response to stress and virulence through PhoP acetylation and deacetylation (Liu et al., 2018). This recent finding should be studied in depth due to the important consequences that it may have on the development of drugs against this disease.

Mycobacterium tuberculosis is a pathogenic bacterium responsible for tuberculosis disease. $M$. tuberculosis is nonetheless present in almost $30 \%$ of the population of the world, although most of them do not develop the disease. A proteomic study carried out by Liu et al. (2014b) showed that lysine deacetylation by Rv1151c, the only sirtuin present in M. tuberculosis, regulated colony morphology, biofilm formation and resistance to heat stress. Interestingly, a strain of M. smegmatis with SrtN overexpressed showed a higher resistance to isoniazid, an anti-tuberculosis drug ( $\mathrm{Gu}$ et al., 2015). INH is supplied as a prodrug that needs to be activated by the catalase peroxidase KatG to couple with $\mathrm{NAD}^{+}$and inhibit the synthesis of mycolic acid, required for $M$. tuberculosis cell wall. SrtN overexpression increases $M$. tuberculosis resistance to INH through KatG down-regulation and intracellular $\mathrm{NAD}^{+}$availability decrease. 


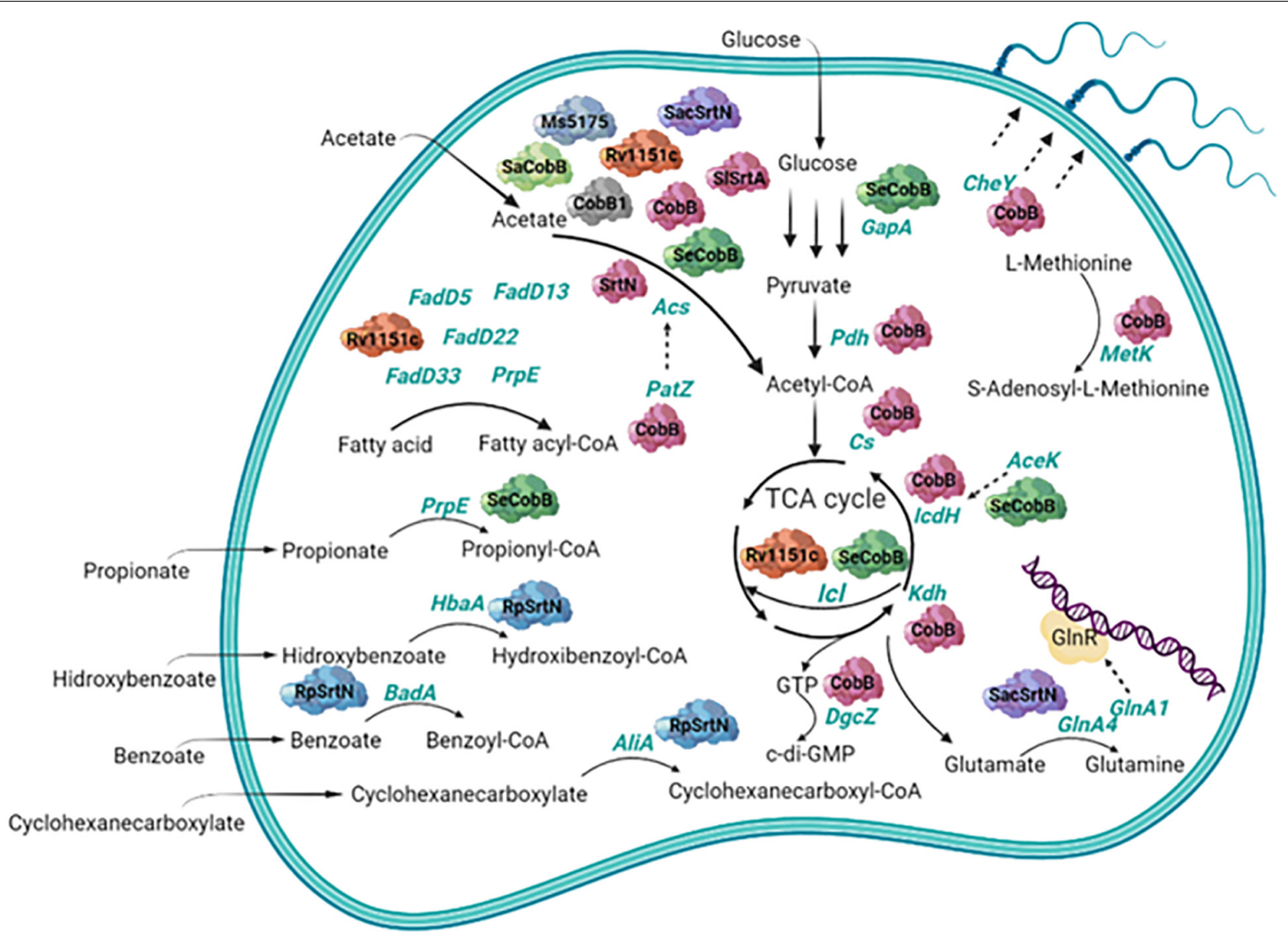

FIGURE 1 | Scheme of the role of bacterial sirtuins in carbon and nitrogen metabolism. Deacetylated substrates are shown in green font and the bacterial sirtuins that deacetylate them have been represented as enzymes of different colors. Sirtuins shown here are represented with the abbreviated names and belong to different species. Correspondence with the full name and species for each one can be found throughout the text.

\section{SIRTUINS IN TRANSCRIPTION AND TRANSLATION PROCESSES}

Regulation of transcription by reversible lysine acetylation has been widely studied in eukaryotes and archaea but not in bacteria. After the first bacterial acetylome studies, several transcription factors were suggested to be regulated by acetylation (Yu et al., 2008; Zhang et al., 2009). E. coli RcsB, a transcription factor involved in cell division and flagellum synthesis, was the first transcription factor identified as CobB substrate in bacteria (Thao et al., 2010). RcsB DNA binding is regulated by the reversible acetylation of the conserved K180 (Thao et al., 2010). K154 was also identified as a target of acetylation and deacetylation by CobB in vivo. K154 acetylation inhibited RcsB while deacetylation by CobB activated it, regulating flagella biosynthesis and motility (Hu et al., 2013; Castaño-Cerezo et al., 2014). The global transcription factor cAMP receptor protein (CRP) has also been reported to be regulated by chemical acetylation, acetylation promoting activity in Class II promoters and ensuring adequate CRP steady state levels in E. coli, although CobB deacetylation has not been demonstrated yet (Écija-Conesa et al., 2020). DnaA is an initiator of replication that plays an essential role in the cell cycle.
A recent study has revealed that the binding of E. coli DnaA to OriC is regulated by acetylation of the two lysines K178 and K243. Deacetylation of DnaA K243 by CobB is necessary for a correct DNA binding (Zhang et al., 2016; Li et al., 2017). A proteomic study carried out recently has identified the global anaerobic regulator FnrL from Rhodobacter sphaeroides (R. sphaeroides) as a substrate of its CobB sirtuin homolog, RsCobB. Thus, acetylation of FnrL decreases its DNA binding ability while the transcription of genes downstream of FnrL increases with deacetylation by RsCobB. This result suggests that reversible lysine acetylation might regulate anaerobic photosynthetic metabolism in this microorganism (Wei et al., 2017).

The first global analysis of the E. coli acetylome also revealed that RNA polymerase can be modified by acetylation. Shortly after, Lime et al. showed that the $\alpha$-subunit of RNAP was regulated by acetylation and deacetylation of its K298. This is specifically required for the stress-responsive cpxP gene transcription (Lima et al., 2011). Gu et al. (2015) identified 27 proteins with an increased level of acetylation in a strain of $M$. smegmatis with the srtN gene deleted. These proteins included the beta subunit of RNA polymerase (rpoC) in addition to metabolic and ribosomal proteins. Other DNA-RNA related 
proteins have also been characterized as sirtuin substrates. Some examples are the proteins $\mathrm{Ku}$ and $\mathrm{LigD}$, components of the non-homologous end-joining (NHEJ) system from M. smegmatis and M. tuberculosis or the E. coli RNAse II (Li et al., 2011; Song et al., 2016). Finally, sirtuins have also been related to translation through E. coli alanyl-tRNA synthetase and Lactobacillus paracasei ribosomal S4 30S protein deacetylation (Atarashi et al., 2016; Torres-Barredo et al., 2018; Umehara et al., 2018). The elongation factor Tu from B. subtilis, TufA, is also regulated by reversible acetylation. Although deacetylation of K42 TufA was catalyzed by the sirtuin SrtN, AcuC Zn-dependent deacetylase also deacetylated TufA, with even greater efficiency (Suzuki et al., 2019).

Sirtuins have emerged as important components of all regulation systems in bacteria, being involved in central metabolism, transcription or virulence regulation. However, the study of the role of sirtuins in bacteria has been limited to a few model organisms, leaving many species unstudied. In addition, even in these model organisms, the degree of knowledge that has been reached is very low, in comparison to eukaryotes. Their functional annotation is limited and further work need to be done to fill in this important gap. Bacterial sirtuins substrates characterized to date are shown in Table 1.

\section{REGULATION OF BACTERIAL SIRTUINS}

The global role demonstrated for bacterial sirtuins suggests a deep regulation of its expression and deacetylase activity at both the transcriptional and post-translational levels. This multidirectional regulation has been demonstrated in human sirtuins, but it is still mostly unknown in bacteria.

Eukaryotic sirtuins exist frequently in different isoforms as a consequence of alternative splicing (Zhang et al., 2021). In 2010, evidence of the existence of sirtuin isoforms in S. enterica were reported. Two active CobB isoforms, a shorter (236 amino acids) and a longer (273 amino acids) ones were studied. Moreover, transcription of the two isoforms started at two different sites (Tucker and Escalante-Semerena, 2010). In E. coli, the existence of these two isoforms has been also demonstrated and it has been suggested that they might be conserved in Enterobacteriae members, excepting species of the Erwinia genus (Umehara et al., 2018).

Regarding the transcriptional regulation of bacterial sirtuins, it has been reported that IolR protein, a regulator of the myoinositol operon, positively controls the transcription of $c o b B$ in $S$. enterica, in addition to the acetyltransferase pat and acs genes (Hentchel et al., 2015). The regulation of sirtuins by different PTMs has been well covered and reported in eukaryotes (Flick and Lüscher, 2012). However, little is known about bacterial sirtuins PTMs. In 2016, a cross-talk between acetylation and phosphorylation was suggested in M. smegmatis and tuberculosis. Mycobacterial sirtuin deacetylase activity seems to be regulated by phosphorylation of a conserved threonine, which is, to date, the only regulation known of a bacterial sirtuin by phosphorylation (Yadav et al., 2016). Also in M. smegmatis, MSMEG_4620 sirtuin has been reported to catalyze its own
ADP-ribosylation (Tan et al., 2015). More recently, regulation of the long isoform of CobB by N-terminal acetylation has been reported and negatively affects its deacetylase activity. Moreover, YiaC, a putative $S$. enterica acetyltransferase has been identified as the responsible for CobB N-terminal acetylation (Parks and Escalante-Semerena, 2020).

Nicotinamide adenine dinucleotide $\left(\mathrm{NAD}^{+}\right)$metabolism is closely related to sirtuins through $\mathrm{NAD}^{+}$consumption and NAM production. Sirtuins activity inhibition by NAM is a global regulation mechanism strongly established (Jackson et al., 2003). The first kinetic study of a bacterial sirtuin was carried out in 2017, and the regulation of CobB in vivo by NAM concentrations was also demonstrated in our work (Gallego-Jara et al., 2017). To identify some E. coli CobB regulators, a study consisting in a proteome microarray identified, in 2014, 183 proteins that bound to CobB (Liu et al., 2014a). A more recent interactome study has revealed that phosphoribosyl pyrophosphate (PRPP) synthetase Prs protein, which was also identified as a potential interactor in 2014, increases CobB deacetylase activity and partially disable its inhibition by nicotinamide (Walter et al., 2020). Prs, is an enzyme responsible for PRPP synthesis, a pivotal metabolite involved, among others, in the $\mathrm{NAD}^{+}$salvage pathway and $\mathrm{NAD}^{+}$de novo biosynthesis. Prs regulation of $\mathrm{CobB}$, might be the nexus between protein acetylation and $\mathrm{NAD}^{+}$metabolism. The ubiquitous regulator cyclic diguanylate, c-di-GMP, also regulates negatively E. coli CobB, inhibiting its deacetylase activity. Moreover, CobB regulates the diguanylate cyclase enzyme, DgcZ, which produces c-di-GMP. CobB deacetylation regulates positively $\mathrm{DgcZ}$, thus increasing DgcZ activity and c-di-GMP level, and establishing a regulator feedback loop (Xu et al., 2019).

\section{PHYLETIC DIVERSITY}

According to Table 1, substrates of 13 bacterial sirtuins (corresponding to 13 species) have been in vitro identified. These organisms represent only three bacterial phyla (Actinobacteria, Firmicutes, and Proteobacteria) and four bacterial classes (Actinomycetia, Bacilli, Alpha-, and Gamma-Proteobacteria) from the 166 phyla and 110 classes defined in the latest releases of the NCBI Taxonomy database (Agarwala et al., 2018). The Proteobacteria class is the most abundant in experimental data and number of species currently sequenced, containing the experimental model species Escherichia coli.

To gain a wider understanding of the diversity of the sirtuin distribution among the full range of phyla in the bacteria superkingdom, we conducted a bioinformatic analysis of the more than 8000 bacterial representative proteomes provided by Uniprot (Bateman et al., 2021). The Uniprot reference proteomes database covers fully annotated proteomes experimentally determined including at least one member of every bacterial phylum in the taxonomic tree (Figure 2). We determined the number of sirtuins encoded in each of the bacterial species searching for the Hidden Markov Model (HMM) profile that corresponds to the sirtuin domain family as defined in the Pfam domain database (Mistry et al., 2021). This 
TABLE 1 | Bacterial sirtuins characterized substrates.

\begin{tabular}{|c|c|c|c|c|c|}
\hline Bacteria & Sirtuin & Substrate & Pathway & Reverted modification & References \\
\hline \multirow[t]{7}{*}{ Salmonella enterica } & SeCobB & Acs & Acetate metabolism & Acetylation & Starai et al., 2002 \\
\hline & SeCobB & PrpE & Propanoate Degradation & Propionylation & Starai et al., 2003 \\
\hline & SeCobB & GapA & Glycolytic process & Acetylation & Wang et al., 2010 \\
\hline & SeCobB & $|c|$ & Glyoxylate cycle & Acetylation & Wang et al., 2010 \\
\hline & SeCobB & AceK & Glycolytic process/glyoxylate cycle & Acetylation & Wang et al., 2010 \\
\hline & SeCobB & PhoP & Transcriptional regulatory protein & Acetylation & Ren et al., 2016 \\
\hline & SeCobB & TacA & Transport & Acetylation & VanDrisse et al., 2017 \\
\hline \multirow[t]{15}{*}{ Escherichia coli } & CobB & Acs & Acetate metabolism & Acetylation & Gallego-Jara et al., 2017 \\
\hline & CobB & CheY & Chemotaxis & Acetylation & Li et al., 2010 \\
\hline & CobB & Cs & Tricarboxylic acid cycle & Acetylation & Venkat et al., 2019 \\
\hline & CobB & $\mathrm{IcdH}$ & Tricarboxylic acid cycle & Acetylation & Venkat et al., 2018 \\
\hline & CobB & NhoA & Post-translational modification & Acetylation & Zhang et al., 2013 \\
\hline & CobB & RNAse ॥ & RNA metabolism & Acetylation & Song et al., 2016 \\
\hline & CobB & MetK & Aspartate superpathway & Acetylation & Sun et al., 2016a \\
\hline & CobB & Alanyl-tRNA synthetase & Translation & Acetylation & Umehara et al., 2018 \\
\hline & CobB & RcsB & Transcription & Acetylation & $\begin{array}{l}\text { Thao et al., 2010; } \\
\text { Castaño-Cerezo et al., } \\
2014\end{array}$ \\
\hline & CobB & RNAP & Transcription & Acetylation & Lima et al., 2011 \\
\hline & CobB & DNAa & Replication & Acetylation & Zhang et al., 2016 \\
\hline & CobB & PatZ & Post-translational modification & Acetylation & $\begin{array}{l}\text { De Diego Puente et al., } \\
2015\end{array}$ \\
\hline & CobB & $\mathrm{PDH}$ & Glycolytic process & Lipoamidation & Rowland et al., 2017 \\
\hline & CobB & $\mathrm{KGH}$ & Tricarboxylic acid cycle & Lipoamidation & Rowland et al., 2017 \\
\hline & CobB & $\mathrm{DgcZ}$ & Purine metabolism & Acetylation & Xu et al., 2019 \\
\hline \multirow[t]{2}{*}{ Bacillus subtilis } & SrtN & AcsA & Acetate metabolism & Acetylation & $\begin{array}{l}\text { Gardner and } \\
\text { Escalante-Semerena, } \\
2009\end{array}$ \\
\hline & SrtN & TufA & Translation & Acetylation & Suzuki et al., 2019 \\
\hline \multirow[t]{5}{*}{ Mycobacterium tuberculosis } & Rv1151c & Acs & Acetate metabolism & Acetylation & Lee et al., 2012 \\
\hline & Rv1151c & $\begin{array}{l}\text { FadD22, FadD2, } \\
\text { FadD5, FadD13 and } \\
\text { FadD33 }\end{array}$ & Fatty acids metabolism & Acetylation & $\begin{array}{l}\text { Nambi et al., 2013; } \\
\text { Vergnolle et al., } 2013\end{array}$ \\
\hline & Rv1151c & lcl & Glyoxylate cycle & Acetylation & Bi et al., 2017 \\
\hline & Rv1151c & $\mathrm{Ku}$ & NHEJ & Acetylation & Li et al., 2011 \\
\hline & Rv1151c & LigD & NHEJ & Acetylation & Li et al., 2011 \\
\hline \multirow[t]{3}{*}{ Mycobacterium smegmatis } & Ms5175 & $\mathrm{Ku}$ & NHEJ & Acetylation & Li et al., 2011 \\
\hline & Ms5175 & LigD & NHEJ & Acetylation & Li et al., 2011 \\
\hline & Ms5175 & Acs & Acetate metabolism & Acetylation & Hayden et al., 2013 \\
\hline Yersinia pestis & YpCobB & PhoP & Transcriptional regulatory protein & Acetylation & Liu et al., 2018 \\
\hline Lactobacillus paracasei & LpSirA & $\begin{array}{l}\text { S4 } 30 \text { S ribosomal } \\
\text { protein }\end{array}$ & Translation & Acetylation & Atarashi et al., 2016 \\
\hline \multirow[t]{3}{*}{ Saccharopolyspora erythraea } & SacSrtN & $\mathrm{G} \ln \mathrm{A} 1$ & Nitrogen metabolism & Acetylation & You et al., 2016 \\
\hline & SacSrtN & GlnA4 & Nitrogen metabolism & Acetylation & You et al., 2016 \\
\hline & SacSrtN & AcsA1, AcsA2, AcsA3 & Acetate metabolism & Acetylation & You et al., 2017 \\
\hline Rhodobacter sphaeroides & RsCobB & FnIR & Anaerobic respiration & Acetylation & Wei et al., 2017 \\
\hline Rhodopseudomonas palustris & RpSrtN & $\begin{array}{l}\text { BadA, HbaA and AliA } \\
\text { synthetases }\end{array}$ & Aromatic metabolism & Acetylation & Crosby et al., 2012 \\
\hline Staphylococcus aureus & SaCobB & Acs & Acetate metabolism & Acetylation & Burckhardt et al., 2019 \\
\hline Streptomyces lividans & SISrtA & Acs and Aacs & Acetate metabolism & Acetylation & $\begin{array}{l}\text { Tucker and } \\
\text { Escalante-Semerena, } \\
\text { 2013; VanDrisse and } \\
\text { Escalante-Semerena, } \\
2018\end{array}$ \\
\hline Streptomyces coelicolor & CobB1 & AcsA & Acetate metabolism & Acetylation & Mikulik et al., 2012 \\
\hline
\end{tabular}

The metabolic role and reverted modification catalyzed by sirtuins is shown. 


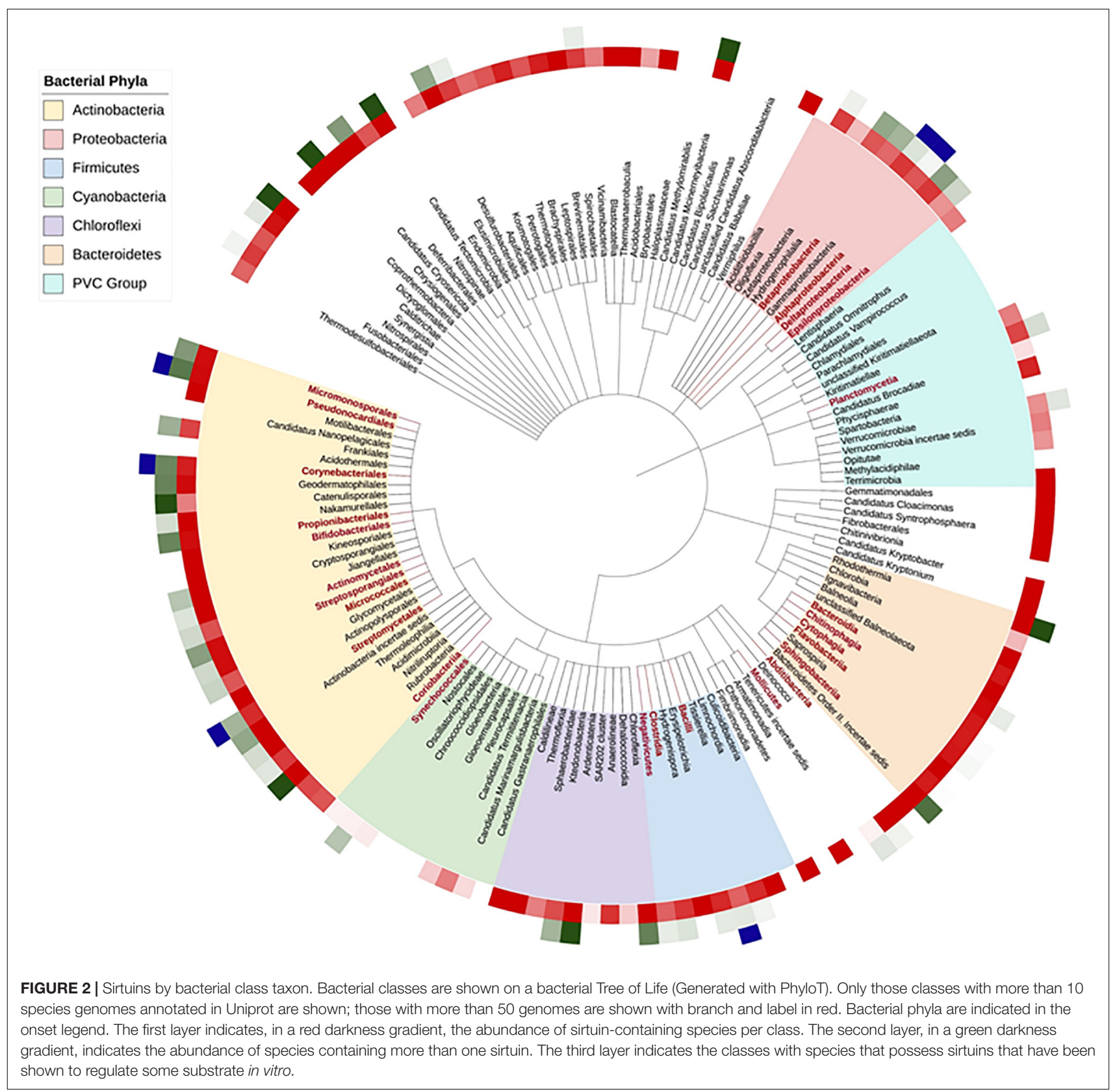

family is coded as SIR2, with the accession number PF02146. A custom-made Python script allowed us to automatize the search and let us localize the number of sirtuins (if any) encoded in each of these reference bacterial genomes. The first layer of Figure 2 shows, in a red darkness gradient, the proportion of sirtuin-containing species with respect to the total number of different species per class, white indicating the absence of sirtuins-containing species in the class. This result indicates that, although sirtuins are widely distributed in prokaryotes and eukaryotes, they are not necessary for the proper growth of some organisms. Interestingly, the sirtuin abundance is not homogeneously distributed among bacterial taxons. As we show on the taxonomic tree pruned to class level, Phyla like Actinobacteria or Firmicutes have a tendency to include at least one sirtuin in their genomes while the majority of the species from the Cyanobacteria/Melainabacteria group lack sirtuins. Moreover, organisms lacking sirtuins perhaps have one or more enzymes that carry out sirtuin roles in metabolism, virulence, or transcription. Finally, it is important to highlight that the number of species and genomes covered by the Uniprot reference proteomes for each class is very disparate. Therefore, for some classes, abundance is the result of hundreds of species average, while other classes are composed of a few species. 
The second layer in Figure 2 shows, in a green darkness gradient, the average number of species in each bacterial class that have at least one sirtuin encoded in its genome, white indicating the species with a single sirtuin. Sirtuins appear mostly as a single unit in bacteria, although an important number of species encoded for two homologous sirtuins. Examples have been found of species containing three or even more sirtuins among the proteins in their proteomes. From the 8002 species analyzed, 2321 do not have a single sirtuin encoded (a 29\%) while 4396 (more than half of the species) have only 1 sirtuin (55\%) and 1132 (14\%) have 2 and only 153 have more than 2. The existence of duplicity in the sirtuin content in single bacterial species has been demonstrated experimentally as has been commented for M. smegmatis in the sirtuins in the carbon and nitrogen metabolism section. There is important heterogeneity in the number of sirtuins encoded by bacterial species even among classes belonging to the same phylum. In Bacteroidetes for example, while almost all classes encode for only one sirtuin, Rhodothermia species show more than three on average. Similar examples of classes with a high number of sirtuins are Ardenticatenia in the Chloroflexi phylum or Nitriliruptoria and Catenulisporales in the Actinobacteria one. Curiously, M. tuberculosis and M. smegmatis, both of them belonging to Actinobacteria phylum, have one and two homologous sirtuins, respectively, and the extra sirtuin from $M$. smegmatis, similar to Sirt4, has been observed in environmental mycobacterial species but not in pathogenic species (Tan et al., 2015).

Finally, as we observe in Figure 2, the number of homologous sirtuins is not directly related to the taxonomy or evolution of the different species, as species more evolved in the taxonomic tree of life can have fewer sirtuins or even none of them than more ancient species.

\section{CLASSIFICATION OF BACTERIAL SIRTUINS}

Based on their phylogenetic relationships, sirtuins are generally grouped into several classes (Frye, 2000; Greiss and Gartner, 2009; Vassilopoulos et al., 2011; Costantini et al., 2013). The majority of these are represented by sirtuins present in human and most vertebrates: SIRT1-SIRT7 (classes I, II, III, and IV). A fifth class (class $\mathrm{U}$ ) includes sirtuins mainly from bacteria and archaea. The last class to date was proposed in 2015, identified in microbial pathogens, and termed SirTMs (Rack et al., 2015).

To classify the sirtuins that have experimental evidence (summarized in Table 1), we generated a multiple sequence alignment [through the Mafft tool with an L-INS-I algorithm (Katoh et al., 2018)]. To the 13 bacterial sirtuins in Table 1, the homologous of these sirtuins were added. Furthermore, to increase the robustness of the analysis, sirtuins from Lactobacillus acidophilus, Bacillus megaterium, Pseudomonas aeruginosa, and Aeromonas hydrophila were also added. These bacteria were selected because the role of their sirtuins has been recently studied (Williams et al., 2012; Ouidir et al., 2015; Olesen et al., 2018; Wang et al., 2020). Altogether, the sequences of 21 sirtuins were aligned (Figure 3B). To assign the classes to the new sequences we included in the alignment a representative of each of the mentioned classes, including the seven human sirtuins. A phylogenetic tree was then built based on the multiple sequence alignment [FastTree with the JTT + CAT substitution model and a Shimodaira-Hasegawa test with 500 replicates for a bootstrap evaluation (Price et al., 2009; Gumerov and Zhulin, 2021)]. This allowed us to observe, even from such a limited set of sirtuins (only the ones with experimental evidence), that there are bacterial representatives for every class of sirtuin previously established from eukaryotes, except for class I (Figure 3A).

As shown in Figure $\mathbf{3 A}$, most of the bacterial sirtuins with experimental evidence, and in particular those sirtuins belonging to the Proteobacteria phylum, have closer common ancestors with the human Sirt5 (class III) than with any other classes. However, there are examples of sirtuins more related to the Sirt4 (class II), with examples from the Actinobacteria phylum. Class IV is more widespread and is present mainly in Streptomyces and class $\mathrm{U}$ in Firmicutes. The most distant common ancestors from the bacterial sirtuins in our selection are those with the human sirtuins Sirt1, Sirt2, and Sirt3, i.e., those belonging to class I. However, sirtuins from the Bacilli genera have ancestors closer related to these class I components than to any other class, which makes a good example of the wide distribution in the classification of the bacterial sirtuins. Firmicutes phylum, to which Bacilli belong, appear in many phylogenetic trees as the older and most divergent group in Bacteria, which could explain the classification of some of their sirtuins in the most distant sirtuin class. Interestingly, bacterial sirtuins belong to different classes, since each of these sirtuin subtypes may be associated with different catalytic preferences as is already demonstrated in the human representative sirtuins. Human Sirt1-3 are considered strong deacetylases, while Sirt5 is mostly associated with other deacylase activities, such as desuccinylase, demalonylase and deglutarylase, and Sirt6 and Sirt4 show strong ADP-ribosyltransferase activity (Michishita et al., 2005; Du et al., 2011; Van Meter et al., 2011; Pannek et al., 2017). Moreover, Sirt6, and also Sirt1, Sirt2, Sirt3, and Sirt5, show deacylation activity against long-chain acyl groups and Sirt2 has lysine debenzoylation activity (Feldman et al., 2013; Huang et al., 2018). It is necessary to increase the number of bacterial sirtuins characterized in vitro to confirm whether this catalytic preference applies to bacterial counterparts. These observations suggest nevertheless a plausible specialization of sirtuins during evolution, allowing the species to adapt to the changing environmental conditions and availability of substrates through the diversification of the functional group they can transfer and the substrate they modify. Further studies on this direction are ongoing through the search for new substrates for sirtuins already characterized and the classification of new sirtuins in different bacterial species from more distant phyla.

Interestingly, those sirtuins that belong to the same species, in the cases where two are encoded, do not necessarily share the same sirtuin class. This is the case for the sirtuins in Pseudomonas aeruginosa (classes III and U), both Streptomyces species (classes II and IV), and Mycobacterium smegmatis (classes II and III). This observation indicates that the emergence of the paralogs in the species holding multiple sirtuin genes in their genomes 

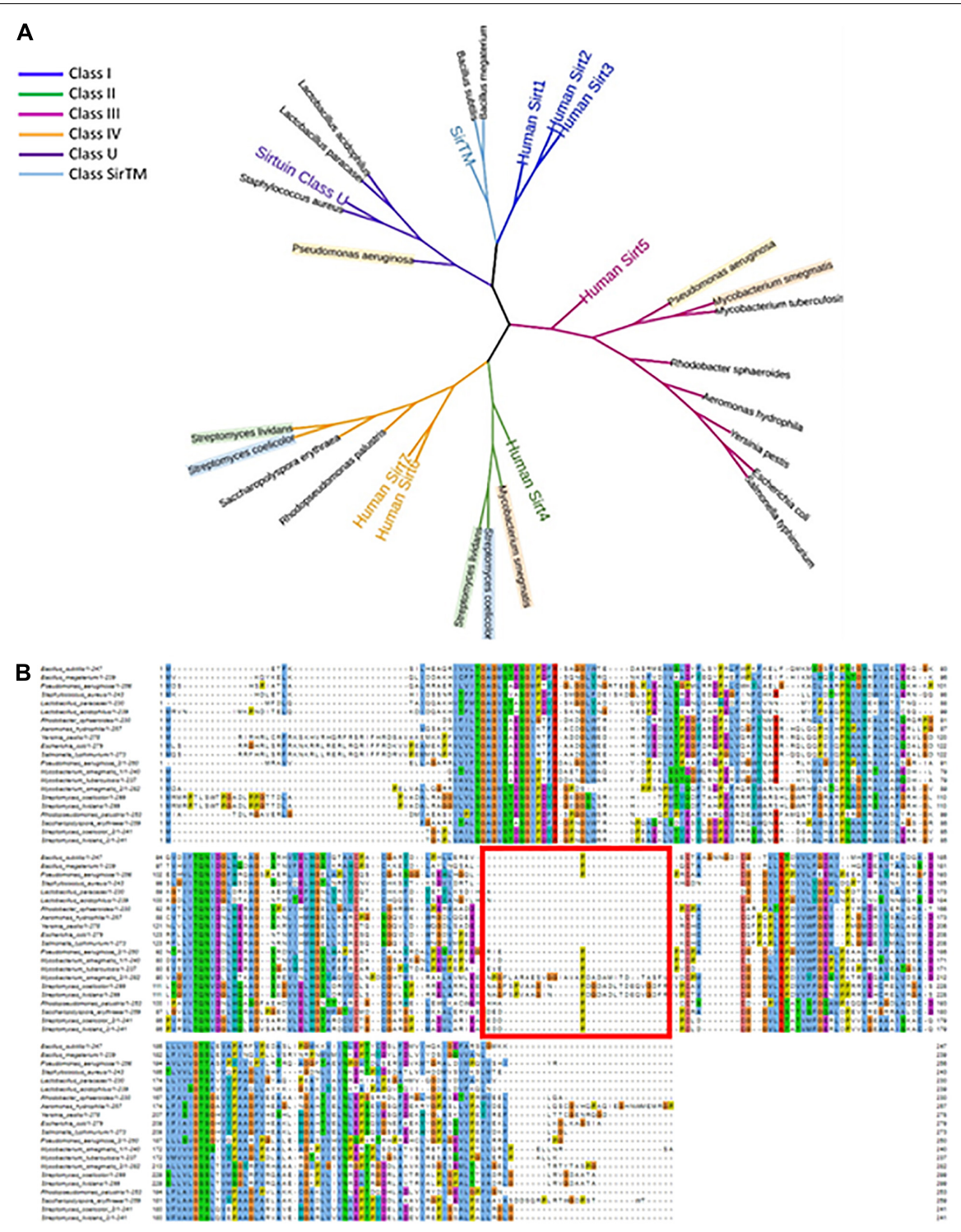

FIGURE 3 | Phylogenetic tree and MSA of the sirtuins covered in this work. (A) Sirtuins are shown labeled with the name of their host species. Those species holding two sirtuins are labeled with a background color. The tree includes all human sirtuins and one representative of sirtuins class $U$ and class $T M$ for class identification. Branch colors indicate the sirtuins class. (B) Mafft multiple sequence alignment of the 21 sirtuins covered in this work. A red square indicates the loop insertion sequence.

is the result of duplication events that occurred in the common ancestors of the majority of the bacterial species summarized in this work. These duplications may have occurred therefore prior to the speciation. It is important to point out that none of the bacterial sirtuins representatives of the class I appear to have a paralog in the genomes of their species. This underscores the fact that this sirtuin class, which is the best studied in human and other vertebrates, is the furthest related with the majority of bacterial sirtuins, as any gene duplication has occurred in those cases posterior to a speciation and have therefore derived into a completely new species.

The results derived above from the classification of the bacterial sirtuins have to be taken with care, however, as they only include those species treated in the present work. Further work is to be done with a wider set of data that should include bacterial sirtuins from a greater number of bacterial species covering the full range of phyla and genera in the tree of life. The set extracted from only 18 species in this work has shown as a good representative of the bacterial sirtuins however and demonstrated that their class distribution is very wide.

Figure 3B shows the multiple sequence alignment that we originally generated for the construction of the phylogenetic tree. It contains the sequences of all the sirtuins treated in this work. It is interesting to point out that sequence lengths are very similar among all the bacterial sirtuins treated here. This is a fact that is not conserved in human or other vertebrate sirtuins, where there 
is a wide variety of lengths, especially in the $\mathrm{C}$ and $\mathrm{N}$ terminal fragments (Costantini et al., 2013). Human or other vertebrate sirtuins contain also a good number of isoforms that are related in a great extent to the $\mathrm{C}$ and $\mathrm{N}$ terminal sections (Szućko, 2016).

The main source of variation among the bacterial sirtuins herein studied comes from a 20-30 amino acid insertion in position 190 (related to Escherichia colis CobB sequence), that is included in one of the two sirtuins from Mycobacterium smegmatis, Streptomyces coelicolor and Streptomyces lividans (all three species from the Actinobacteria phylum, Figure 3B). This small insertion appears to be located between the helices $\alpha 8$ and $\alpha 9$ as shown in the sirtuin 3D structure (Cosgrove et al., 2006; Yuan and Marmorstein, 2012). These two regions are involved in the binding pocket of $\mathrm{Zn}^{2+}$, which plays a structural role. The loop insertion appears to be a common feature that is exclusive of class II sirtuins and has been previously observed in human Sirt4 (Pannek et al., 2017). It could play an important role in catalysis regulation, contributing to substrate binding and restricting active site dynamics. A larger investigation with the full set of reference proteomes from Uniprot is currently ongoing to further confirm the importance of this insertion and other features differentiating all bacterial sirtuins among them and with the known eukaryotic sirtuins.

\section{CONCLUSION}

For years, sirtuins were only related to transcription regulation in eukaryotes by deacetylation of histones. Nowadays, it is known that they are involved in many metabolic pathways in addition to transcription regulation. Sirtuins have been widely studied in humans, where they regulate important metabolic pathways. However, little is known about sirtuins in lower organisms: only 13 bacterial sirtuins have been studied in vitro from the 8000 bacterial representative proteomes provided by Uniprot. With only this small sample a great diversity has been suggested both in the number of sirtuins per species and in the type of sirtuin. The phylogenetic study carried out in the last part of this review reveals the evolutionary differences between sirtuins of different bacterial species and even between homologous sirtuins. Future studies will be necessary to understand the diversity that emerged during evolution and thus to increase the knowledge about this important family of enzymes.

\section{REFERENCES}

AbouElfetouh, A., Kuhn, M. L., Hu, L. I., Scholle, M. D., Sorensen, D. J., Sahu, A. K., et al. (2015). The E. coli sirtuin CobB shows no preference for enzymatic and nonenzymatic lysine acetylation substrate sites. Microbiologyopen 4, 66-83. doi: $10.1002 / \mathrm{mbo} 3.223$

Agarwala, R., Barrett, T., Beck, J., Benson, D. A., Bollin, C., Bolton, E., et al. (2018). Database resources of the National Center for Biotechnology Information. Nucleic Acids Res. 46, D8-D13. doi: 10.1093/nar/gkx 1095

Appel, C. D., Feld, G. K., Wallace, B. D., and Williams, R. S. (2016). Structure of the sirtuin-linked macrodomain SAV0325 from Staphylococcus aureus. Protein Sci. 25, 1682-1691. doi: 10.1002/pro.2974
On the other hand, it is essential to continue investigating the stoichiometry of deacetylation by sirtuins to know if the low stoichiometry reported for E. colis CobB is conserved in other organisms and to know its physiological meaning. Furthermore, the low number of targeted proteins whose activity is regulated by sirtuins deacetylation identified to date contrasts to the high number of proteins that have been identified as their substrates in proteomic studies (Castaño-Cerezo et al., 2014; AbouElfetouh et al., 2015; Weinert et al., 2017). It seems difficult that the activity of all of them is regulated by sirtuin-mediated deacetylation, since the physiological behavior of the sirtuin-deficient strains is similar to that of the wild type. Some studies suggest that the sirtuins regulatory role might be more important in stressful or nutrient limited conditions, when acetylation, especially nonenzymatic is higher than in non-stressful situations (Schilling et al., 2015, 2019; Weinert et al., 2017).

Research in the field of bacterial sirtuins is essential to expand our knowledge about molecular signaling pathways, gene expression regulation, and associated physiological functions in prokaryotic organisms, which are fundamental for the advancement of fields such as synthetic biology or pharmacology.

\section{AUTHOR CONTRIBUTIONS}

JG-J, ÁO, and TD contributed to the conceptualization. JG-J and ÁO contributed to the writing-original draft preparation. JG-J, ÁO, GL, RS, MC, and TD contributed to the writing-review and editing. TD contributed to the project administration. TD contributed to the funding acquisition. All authors have read and agreed to the published version of the manuscript.

\section{FUNDING}

This work was supported by Grant RTI2018-094393-B-C21 funded by MCIN/AEI/ 10.13039/501100011033 and by "ESF A way of making Europe" and the Seneca Foundation Science and Technology Agency for the Region of Murcia (20786/PI/18). GL is a recipient of a Ph.D. fellowship from Seneca Foundation (20715/FPI/18) and RS is a recipient of an FPUPh. D. fellowship from the Ministry of Science, Innovation and Universities (FPU18/00545).

Atarashi, H., Kawasaki, S., Niimura, Y., Tanaka, N., Okada, S., Shiwa, Y., et al. (2016). Identification of sirtuin and its target as the ribosomal protein S4 in Lactobacillus paracasei. J. Gen. Appl. Microbiol. 62, 98-105. doi: 10.2323/jgam. 62.98

Avalos, J. L., Celic, I., Muhammad, S., Cosgrove, M. S., Boeke, J. D., and Wolberger, C. (2002). Structure of a Sir2 enzyme bound to an acetylated p53 peptide. Mol. Cell 10, 523-535. doi: 10.1016/s1097-2765(02)00628-627

Baeza, J., Dowell, J. A., Smallegan, M. J., Fan, J., Amador-Noguez, D., Khan, Z., et al. (2014). Stoichiometry of site-specific lysine acetylation in an entire proteome. J. Biol. Chem. 289, 21326-21338. doi: 10.1074/jbc.M114.581843

Bateman, A., Martin, M. J., Orchard, S., Magrane, M., Agivetova, R., Ahmad, S., et al. (2021). UniProt: the universal protein knowledgebase in 2021. Nucleic Acids Res. 49, D480-D489. doi: 10.1093/nar/gkaa1100 
Bernal, V., Castaño-Cerezo, S., Gallego-Jara, J., Écija-Conesa, A., de Diego, T., Iborra, J. L., et al. (2014). Regulation of bacterial physiology by lysine acetylation of proteins. N. Biotechnol. 31, 586-595. doi: 10.1016/j.nbt.2014.03.002

Bi, J., Wang, Y., Yu, H., Qian, X., Wang, H., Liu, J., et al. (2017). Modulation of central carbon metabolism by acetylation of isocitrate lyase in Mycobacterium tuberculosis. Sci. Rep. 7:44826. doi: 10.1038/srep44826

Brachmann, C. B., Sherman, J. M., Devine, S. E., Cameron, E. E., Pillus, L., and Boeke, J. D. (1995). The SIR2 gene family, conserved from bacteria to humans, functions in silencing, cell cycle progression, and chromosome stability. Genes Dev. 9, 2888-2902. doi: 10.1101/gad.9.23.2888

Burckhardt, R. M., Buckner, B. A., and Escalante-Semerena, J. C. (2019). Staphylococcus aureus modulates the activity of acetyl- Coenzyme A synthetase (Acs) by sirtuin-dependent reversible lysine acetylation. Mol. Microbiol. 112, 588-604. doi: 10.1111/mmi.14276.Staphylococcus

Carabetta, V. J., and Cristea, M. (2017). Regulation, function, and detection of protein acetylation in bacteria. J. Bacteriol. 199:e00107-17. doi: 10.1128/JB. 00107- 17

Castaño-Cerezo, S., Bernal, V., Post, H., Fuhrer, T., Cappadona, S., Sanchez-Diaz, N., et al. (2014). Protein acetylation affects acetate metabolism, motility and acid stress response in Escherichia coli. Mol. Syst. Biol. 10:762. doi: 10.15252/msb. 20145227

Cheng, Z., Tang, Y., Chen, Y., Kim, S., Liu, H., Shawn, S. C., et al. (2009). Molecular characterization of propionyllysines in non-histone proteins. Mol. Cell. Proteomics 8, 45-52. doi: 10.1074/mcp.M800224-MCP200

Christensen, D. G., Baumgartner, J. T., Xie, X., Jew, K. M., Basisty, N., and Schilling, B. (2019). Mechanisms, detection, and relevance of protein acetylation in prokaryotes. mBio 10:e2708-18. doi: 10.1128/mBio.02708-18

Christensen, D. G., Meyer, J. G., Baumgartner, J. T., D'Souza, A. K., Nelson, W. C., Payne, S. H., et al. (2018). Identification of novel protein lysine acetyltransferases in Escherichia coli. mBio 9:e01905-18. doi: 10.1128/mBio. 01905- 1918

Colak, G., Xie, Z., Zhu, A. Y., Dai, L., Lu, Z., Zhang, Y., et al. (2013). Identification of lysine succinylation substrates and the succinylation regulatory enzyme CobB in Escherichia coli. Mol. Cell. Proteomics 12, 3509-3520. doi: 10.1016/j.physa. 2016.12.079

Cosgrove, M. S., Bever, K., Avalos, J. L., Muhammad, S., Zhang, X., and Wolberger, C. (2006). The structural basis of sirtuin substrate affinity. Biochemistry 45, 7511-7521. doi: 10.1021/bi0526332

Costantini, S., Sharma, A., Raucci, R., Costantini, M., Autiero, I., and Colonna, G. (2013). Genealogy of an ancient protein family: the Sirtuins, a family of disordered members. BMC Evol. Biol. 13:60. doi: 10.1186/1471-2148$13-60$

Crosby, H. A., and Escalante-Semerena, J. C. (2014). The acetylation motif in AMPforming acyl coenzyme a synthetases contains residues critical for acetylation and recognition by the protein acetyltransferase pat of Rhodopseudomonas palustris. J. Bacteriol. 196, 1496-1504. doi: 10.1128/JB.00004- 14

Crosby, H. A., Heiniger, E. K., Harwood, C. S., and Escalante-Semerena, J. C. (2010). Reversible N-Lysine acetylation regulates the activity of acyl-CoA synthetases involved in anaerobic benzoate catabolism in Rhodopseudomonas palustris. Mol. Microbiol. 76, 874-888. doi: 10.1111/j.1365-2958.2010. 07127

Crosby, H. A., Pelletier, D. A., Hurst, G. B., and Escalante-Semerena, J. C. (2012). System-wide studies of N-Lysine acetylation in Rhodopseudomonas palustris reveals substrate specificity of protein acetyltransferases. J. Biol. Chem. 287, 15590-15601. doi: 10.1074/jbc.M112.352104

De Diego Puente, T., Gallego-Jara, J., Castaño-Cerezo, S., Sánchez, V. B., Espín, V. F., De La Torre, J. G., et al. (2015). The protein acetyltransferase PatZ from Escherichia coli is regulated by autoacetylation-induced oligomerization. J. Biol. Chem. 290, 23077-23093. doi: 10.1074/jbc.M115.649806

Drazic, A., Myklebust, L. M., Ree, R., and Arnesen, T. (2016). The world of protein acetylation. Biochim. Biophys. Acta - Proteins Proteomics 1864, 1372-1401. doi: 10.1016/j.bbapap.2016.06.007

Du, J., Zhou, Y., Su, X., Yu, J. J., Khan, S., Jiang, H., et al. (2011). Sirt5 is a NAD-dependent protein lysine demalonylase and desuccinylase. Science 334, 806-809. doi: 10.1126/science. 1207861

Écija-Conesa, A., Gallego-Jara, J., Terol, G. L., Browning, D. F., Busby, S. J. W., Wolfe, A. J., et al. (2020). An ideal spacing is required for the control of Class
II CRP-dependent promoters by the status of CRP K100. FEMS Microbiol. Lett. 367:fnaa164. doi: 10.1093/FEMSLE/FNAA164

Feldman, J. L., Baeza, J., and Denu, J. M. (2013). Activation of the protein deacetylase SIRT6 by long-chain fatty acids and widespread deacylation by mammalian sirtuins. J. Biol. Chem. 288, 31350-31356. doi: 10.1074/jbc.C113. 511261

Flick, F., and Lüscher, B. (2012). Regulation of sirtuin function by posttranslational modifications. Front. Pharmacol. 3:29. doi: 10.3389/fphar.2012.00029

Frye, R. A. (2000). Phylogenetic classification of prokaryotic and eukaryotic Sir2like proteins. Biochem. Biophys. Res. Commun. 273, 793-798. doi: 10.1006/bbrc. 2000.3000

Gallego-Jara, J., Écija Conesa, A., de Diego Puente, T., Lozano Terol, G., and Cánovas Díaz, M. (2017). Characterization of CobB kinetics and inhibition by nicotinamide. PLoS One 12:e189689. doi: 10.1371/journal.pone.0189689

Gardner, J. G., and Escalante-Semerena, J. C. (2009). In Bacillus subtilis, the sirtuin protein deacetylase, encoded by the srtN gene (formerly yhdZ), and functions encoded by the acuABC genes control the activity of acetyl coenzyme a synthetase. J. Bacteriol. 191, 1749-1755.

Gardner, J. G., Grundy, F. J., Henkin, T. M., and Escalante-Semerena, J. C. (2006). Control of acetyl-Coenzyme a synthetase (AcsA) activity by acetylation/deacetylation without NAD+ involvement in Bacillus subtilis. J. Bacteriol. 188, 5460-5468. doi: 10.1128/JB.00215-216

Garrity, J., Gardner, J. G., Hawse, W., Wolberger, C., and Escalante-Semerena, J. C. (2007). N-lysine propionylation controls the activity of propionyl-CoA synthetase. J. Biol. Chem. 282, 30239-30245. doi: 10.1074/jbc.M704409200

Gil, J., Ramírez-Torres, A., Chiappe, D., Luna-Penãloza, J., Fernandez-Reyes, F. C., Arcos-Encarnación, B., et al. (2017). Lysine acetylation stoichiometry and proteomics analyses reveal pathways regulated by sirtuin 1 in human cells. J. Biol. Chem. 292, 18129-18144. doi: 10.1074/jbc.M117.784546

Gregoretti, I. V., Lee, Y.-M., and Goodson, H. V. (2004). Molecular evolution of the histone deacetylase family: functional implications of phylogenetic analysis. J. Mol. Biol. 338, 17-31. doi: 10.1016/j.jmb.2004.02.006

Greiss, S., and Gartner, A. (2009). Sirtuin/Sir2 phylogeny, evolutionary considerations and structural conservation. Mol. Cells 28, 407-415. doi: 10. 1007/s10059-009-0169-x

Grundy, F. J., Waters, D. A., Tsetska, Y., and Henkin, T. M. (1993). Identification of genes involved in utilization of acetate and acetoin in Bacillus subtilis. Mol. Microbiol. 10, 259-271.

Gu, L., Chen, Y., Wang, Q., Li, X., Mi, K., and Deng, H. (2015). Functional characterization of sirtuin-like protein in Mycobacterium smegmatis. J Proteome Res. 14, 4441-4449. doi: 10.1021/acs.jproteome.5b00359

Gumerov, V. M., and Zhulin, I. B. (2021). TREND: a platform for exploring protein function in prokaryotes based on phylogenetic, domain architecture and gene neighborhood analyses. Nucleic Acids Res. 48, W72-W76. doi: 10.1093/NAR/ GKAA243

Han, X., Shen, L., Wang, Q., Cen, X., Wang, J., Wu, M., et al. (2017). Cyclic AMP inhibits the activity and promotes the acetylation of acetyl-CoA synthetase through competitive binding to the ATP/AMP pocket. J. Biol. Chem. 292, 1374-1384. doi: 10.1074/jbc.M116.753640

Hayden, J. D., Brown, L. R., Gunawardena, H. P., Perkowski, E. F., Chen, X., and Braunstein, M. (2013). Reversible acetylation regulates acetate and propionate metabolism in Mycobacterium smegmatis. Microbiology 159, 1986-1999. doi: 10.1099/mic.0.068585-68580

Hebbes, T. R., Thorne, A. W., and Crane-Robinson, C. (1988). A direct link between core histone acetylation and transcriptionally active chromatin. EMBO J. 7, 1395-1402. doi: 10.1002/j.1460-2075.1988.tb02956.x

Hentchel, K. L., Thao, S., Intile, P. J., and Escalante-Semerena, J. C. (2015). Deciphering the regulatory circuitry that controls reversible lysine acetylation in Salmonella enterica. mBio 6:e00891-15. doi: 10.1128/mBio.008 91-815

Hu, L. I., Chi, B. K., Kuhn, M. L., Filippova, E. V., Walker-Peddakotla, A. J., Bäsell, K., et al. (2013). Acetylation of the response regulator RcsB controls transcription from a small RNA promoter. J. Bacteriol. 195, 4174-4186. doi: 10.1128/JB.00383-313

Huang, H., Zhang, D., Wang, Y., Perez-Neut, M., Han, Z., Zheng, Y. G., et al. (2018). Lysine benzoylation is a histone mark regulated by SIRT2. Nat. Commun. 9:3374. doi: 10.1038/s41467-018-05567-w 
Imai, S., Armstrong, C. M., Kaeberlein, M., and Guarente, L. (2000). Transcriptional silencing and longevity protein Sir2 is an NAD-dependent histone deacetylase. Lett. Nat. 403, 796-800.

Ivy, J. M., Klar, A. J., and Hicks, J. B. (1986). Cloning and characterization of four SIR genes of Saccharomyces cerevisiae. Mol. Cell. Biol. 6, 688-702. doi: $10.1128 / \mathrm{mcb} .6 .2 .688$

Jackson, M. D., Schmidt, M. T., Oppenheimer, N. J., and Denu, J. M. (2003). Mechanism of nicotinamide inhibition and transglycosidation by Sir2 histone/protein deacetylases. J. Biol. Chem. 278, 50985-50998. doi: 10.1074/jbc. M306552200

James, A. M., Hoogewijs, K., Logan, A., Hall, A. R., Ding, S., Fearnley, I. M., et al. (2017). Non-enzymatic N-acetylation of lysine residues by acetyl-CoA often occurs via a proximal S-acetylated thiol intermediate sensitive to glyoxalase II. Cell Rep. 18, 2105-2112. doi: 10.1016/j.celrep.2017.02.018

Kaeberlein, M., McVey, M., and Guarente, L. (1999). The SIR2/3/4 complex and SIR2 alone promote longevity in Saccharomyces cerevisiae by two different mechanisms. Genes Dev. 13, 2570-2580. doi: 10.1101/gad.13.19.2570

Katoh, K., Rozewicki, J., and Yamada, K. D. (2018). MAFFT online service: multiple sequence alignment, interactive sequence choice and visualization. Brief. Bioinform. 20, 1160-1166. doi: 10.1093/bib/bbx108

Kennedy, B. K., Austriaco, N. R., Zhang, J., and Guarente, L. (1995). Mutation in the silencing gene S/R4 can delay aging in S. cerevisiae. Cell 80, 485-496. doi: 10.1016/0092-8674(95)90499-90499

Kim, D., Yu, B. J., Kim, J. A., Lee, Y. J., Choi, S. G., Kang, S., et al. (2013). The acetylproteome of Gram-positive model bacterium Bacillus subtilis. Proteomics 13, 1726-1736. doi: 10.1002/pmic.201200001

Klar, A. J. S., Fogel, S., and Macleod, K. (1979). Mar1 - a regulator of the HM $\alpha$ and $\mathrm{HM} \alpha$ loci in Saccharomyces cerevisiae. Genetics 93, 37-50.

Kosono, S., Tamura, M., Suzuki, S., Kawamura, Y., Yoshida, A., Nishiyama, M., et al. (2015). Changes in the acetylome and succinylome of Bacillus subtilis in response to carbon source. PLoS One 10:e0131169. doi: 10.1371/journal.pone. 0131169

Kuhn, M. L., Zemaitaitis, B., Hu, L. I., Sahu, A., Sorensen, D., Minasov, G., et al. (2014). Structural, kinetic and proteomic characterization of Acetyl phosphatedependent bacterial protein acetylation. PLoS One 9:e94816. doi: 10.1371/ journal.pone.0094816

Lee, H. J., Lang, P. T., Fortune, S. M., Sassetti, C. M., and Alber, T. (2012). Cyclic AMP regulation of protein lysine acetylation in Mycobacterium tuberculosis. Nat. Struct. Mol. Biol. 19, 811-818. doi: 10.1038/nsmb.2318

Li, L., Wang, W., Zhang, R., Xu, J., Wang, R., Wang, L., et al. (2018). First acetylproteome profiling of Salmonella Typhimurium revealed involvement of lysine acetylation in drug resistance. Vet. Microbiol. 226, 1-8. doi: 10.1016/j.vetmic. 2018.09.024

Li, R., Gu, J., Chen, Y. Y., Xiao, C., Le, Wang, L. W., et al. (2010). CobB regulates Escherichia coli chemotaxis by deacetylating the response regulator CheY. Mol. Microbiol. 76, 1162-1174. doi: 10.1111/j.1365-2958.2010.07125.x

Li, S., Zhang, Q., Xu, Z., and Yao, Y. F. (2017). Acetylation of lysine 243 inhibits the oriC binding ability of DnaA in Escherichia coli. Front. Microbiol. 8:699. doi: $10.3389 /$ fmicb.2017.00699

Li, Z., Wen, J., Lin, Y., Wang, S., Xue, P., Zhang, Z., et al. (2011). A sir2-like protein participates in mycobacterial NHEJ. PLoS One 6:e20045. doi: 10.1371/journal. pone.0020045

Lima, B. P., Antelmann, H., Katrin, G., Khanh Chi, B., Becher, D., Brinsmade, S. R., et al. (2011). Involvement of protein acetylation in glucose-induced transcription of a stress-responsive promoter. Mol. Microbiol. 81, 1190-1204. doi: 10.1111/j.1365-2958.2011.07742.x

Liu, F., Yang, M., Wang, X., Yang, S., Gu, J., Zhou, J., et al. (2014b). Acetylome analysis reveals diverse functions of lysine acetylation in Mycobacterium tuberculosis. Mol. Cell. Proteomics 13, 3352-3366. doi: 10.1074/mcp.M114. 041962

Liu, C. X., Wu, F. L., Jiang, H. W., He, X., Guo, S. J., and Tao, S. C. (2014a). Global identification of CobB interactors by an Escherichia coli proteome microarray. Acta Biochim. Biophys. Sin. (Shanghai) 46, 548-555. doi: 10.1093/abbs/ gmu038

Liu, W., Tan, Y., Cao, S., Zhao, H., Fang, H., Yang, X., et al. (2018). Protein acetylation mediated by YfiQ and CobB is involved in the virulence and stress response of Yersinia pestis. Infect. Immun. 86:e00224-18. doi: 10.1128/IAI. 00224-218
Ma, Q., and Wood, T. K. (2011). Protein acetylation in procaryotes increases stress resistance. Biochem. Biophys. Res. Commun. 410, 846-851. doi: 10.1016/j.bbrc. 2011.06.076.Protein

Macek, B., Forchhammer, K., Hardouin, J., Weber-Ban, E., Grangeasse, C., and Mijakovic, I. (2019). Protein post-translational modifications in bacteria. Nat. Rev. Microbiol. 17, 651-664. doi: 10.1038/s41579-019-0243-240

Mathias, R. A., Greco, T. M., Oberstein, A., Budayeva, H. G., Chakrabarti, R., Rowland, E. A., et al. (2014). Sirtuin 4 is a lipoamidase regulating pyruvate dehydrogenase complex activity. Cell 159, 1615-1625. doi: 10.1016/j.cell.2014. 11.046

Mei, X. Y., He, X., Di, Huang, L., Qi, D. S., Nie, J., et al. (2016). Dehomocysteinylation is catalysed by the sirtuin-2-like bacterial lysine deacetylase CobB. FEBS J. 283, 4149-4162. doi: 10.1111/febs.13912

Meyer, J. G., D'Souza, A. K., Sorensen, D. J., Rardin, M. J., Wolfe, A. J., Gibson, B. W., et al. (2016). Quantification of lysine acetylation and succinylation stoichiometry in proteins using mass spectrometric dataindependent acquisitions (SWATH). J. Am. Soc. Mass Spectrom. 27, 1758-1771. doi: 10.1007/s13361-016-1476-z

Michishita, E., Park, J. Y., Burneskis, J. M., and Barrett, J. C. (2005). Evolutionarily conserved and nonconserved cellular localizations and functions of human SIRT proteins. Mol. Biol Cell 16, 4623-4635. doi: 10.1091/mbc.E05

Mikulik, K., Felsberg, J., Kudrnáčová, E., Bezoušková, S., Šetinová, D., Stodůlková, E., et al. (2012). CobB1 deacetylase activity in Streptomyces coelicolor. Biochem. Cell Biol. 90, 179-187. doi: 10.1139/o11-086

Mistry, J., Chuguransky, S., Williams, L., Qureshi, M., Salazar, G. A., Sonnhammer, E. L. L., et al. (2021). Pfam: the protein families database in 2021. Nucleic Acids Res. 49, D412-D419. doi: 10.1093/nar/gkaa913

Nakayasu, E. S., Burnet, M. C., Walukiewicz, H. E., Wilkins, C. S., Shukla, A. K., Brooks, S., et al. (2017). Ancient regulatory role of lysine acetylation in central metabolism. mBio 8:e01894-17. doi: 10.1128/mBio.01894-17

Nambi, S., Gupta, K., Bhattacharya, M., Ramakrishnan, P., Ravikumar, V., Siddiqui, N., et al. (2013). Cyclic AMP-dependent protein lysine acylation in Mycobacteria regulates fatty acid and propionate metabolism. J. Biol. Chem. 288, 14114-14124. doi: 10.1074/jbc.M113.463992

Nguyen, K. T., Mun, S. H., Lee, C. S., and Hwang, C. S. (2018). Control of protein degradation by $\mathrm{N}$-terminal acetylation and the $\mathrm{N}$-end rule pathway. Exp. Mol. Med. 50, 1-8. doi: 10.1038/s12276-018-0097-y

Nurulain, T., and Zaveri (2016). Bacterial protein acetylation: new discoveries unanswered questions. Physiol. Behav. 176, 139-148. doi: 10.1007/s00294-0150552-554

Olesen, S. V., Rajabi, N., Svensson, B., Olsen, C. A., and Madsen, A. S. (2018). An NAD+-Dependent sirtuin depropionylase and deacetylase (Sir2La) from the probiotic bacterium Lactobacillus acidophilus NCFM. Biochemistry 57, 3903-3915. doi: 10.1021/acs.biochem.8b00306

Olsen, J. V., and Mann, M. (2013). Status of large-scale analysis of posttranslational modifications by mass spectrometry. Mol. Cell. Proteomics 12, 3444-3452. doi: 10.1074/mcp.O113.034181

Ouidir, T., Cosette, P., Jouenne, T., and Hardouin, J. (2015). Proteomic profiling of lysine acetylation in Pseudomonas aeruginosa reveals the diversity of acetylated proteins. Proteomics 15, 2152-2157. doi: 10.1002/pmic. 201500056

Pannek, M., Simic, Z., Fuszard, M., Meleshin, M., Rotili, D., Mai, A., et al. (2017). Crystal structures of the mitochondrial deacylase Sirtuin 4 reveal isoformspecific acyl recognition and regulation features. Nat. Commun. 8:1513. doi: 10.1038/s41467-017-01701-1702

Parks, A. R., and Escalante-Semerena, J. C. (2020). Modulation of the bacterial CobB sirtuin deacylase activity by N-terminal acetylation. Proc. Natl. Acad. Sci. U S A. 117, 15895-15901. doi: 10.1073/PNAS.2005296117

Price, M. N., Dehal, P. S., and Arkin, A. P. (2009). Fasttree: computing large minimum evolution trees with profiles instead of a distance matrix. Mol. Biol. Evol. 26, 1641-1650. doi: 10.1093/molbev/msp077

Rack, J. G. M., Morra, R., Barkauskaite, E., Kraehenbuehl, R., Ariza, A., Qu, Y., et al. (2015). Identification of a class of protein ADP-Ribosylating sirtuins in microbial pathogens. Mol. Cell 59, 309-320. doi: 10.1016/j.molcel.2015.06.013

Reed, L. J. (2001). A trail of research from lipoic acid to $\alpha$-Keto acid dehydrogenase complexes. J. Biol. Chem. 276, 38329-38336. doi: 10.1074/jbc.R100026200

Reed, L. J., Koike, M., Levitch, M. E., and Leach, F. R. (1958). Studies on the nature and reactions of protein-bound lipoic acid. J. Biol. Chem. 232, 143-158. doi: 10.1016/s0021-9258(18)70382-70387 
Ren, J., Sang, Y., Ni, J., Tao, J., Lu, J., Zhao, M., et al. (2015). Acetylation regulates survival of Salmonella enterica serovar typhimurium under acid stress. Appl. Environ. Microbiol. 81, 5675-5682. doi: 10.1128/AEM.01009-1015

Ren, J., Sang, Y., Tan, Y., Tao, J., Ni, J., Liu, S., et al. (2016). Acetylation of Lysine 201 inhibits the DNA-Binding ability of PhoP to regulate Salmonella virulence. PLoS Pathog. 12:e1005458. doi: 10.1371/journal.ppat.1005458

Rowland, E. A., Greco, T. M., Snowden, C. K., McCabe, A. L., Silhavy, T. J., and Cristea, I. M. (2017). Sirtuin lipoamidase activity is conserved in bacteria as a regulator of metabolic enzyme complexes. mBio 8:e01096-17. doi: 10.1128/ mBio.01096-1017

Schilling, B., Basisty, N., Christensen, D. G., Sorensen, D., Orr, J. S., Wolfe, A. J., et al. (2019). Global lysine acetylation in Escherichia coli results from growth conditions that favor acetate fermentation. J. Bacteriol. 201:e00768-18. doi: 10.1128/JB.00768-18

Schilling, B., Christensen, D., Davis, R., Sahu, A. K., Hu, L. I., Walker-Peddakotla, A., et al. (2015). Protein acetylation dynamics in response to carbon overflow in Escherichia coli. Mol. Microbiol. 98, 847-863. doi: 10.1111/mmi.13161

Schwer, B., Bunkenborg, J., Verdin, R. O., Andersen, J. S., and Verdin, E. (2006). Reversible lysine acetylation controls the activity of the mitochondrial enzyme acetyl-CoA synthetase 2. Proc. Natl. Acad. Sci. U S A. 103, 10224-10229. doi: 10.1073/pnas.0603968103

Seidel, J., Klockenbusch, C., and Schwarzer, D. (2016). Investigating deformylase and deacylase activity of mammalian and bacterial sirtuins. ChemBioChem 17, 398-402. doi: 10.1002/cbic.201500611

Song, L., Wang, G., Malhotra, A., Deutscher, M. P., and Liang, W. (2016). Reversible acetylation on Lys501 regulates the activity of RNase II. Nucleic Acids Res. 44, 1979-1988. doi: 10.1093/nar/gkw053

Spange, S., Wagner, T., Heinzel, T., and Krämer, O. H. (2009). Acetylation of nonhistone proteins modulates cellular signalling at multiple levels. Int. J. Biochem. Cell Biol. 41, 185-198. doi: 10.1016/j.biocel.2008.08.027

Starai, V. J., Celic, I., Cole, R. N., Boeke, J. D., and Escalante-Semerena, J. C. (2002). Sir2-dependent activation of acetyl-CoA synthetase by deacetylation of active lysine. Science 298, 2390-2392. doi: 10.1126/science.1077650

Starai, V. J., Takahashi, H., Boeke, J. D., and Escalante-Semerena, J. C. (2003). Short-chain fatty acid activation by acyl-coenzyme a synthetases requires SIR2 protein function in Salmonella enterica and Saccharomyces cerevisiae. Genetics $163,545-555$.

Sun, M., Xu, J., Wu, Z., Zhai, L., Liu, C., Cheng, Z., et al. (2016b). Characterization of protein lysine propionylation in Escherichia coli: global profiling, dynamic change, and enzymatic regulation. J. Proteome Res. 15, 4696-4708. doi: 10.1021/ acs.jproteome.6b00798

Sun, M., Guo, H., Lu, G., Gu, J., Wang, X., Zhang, X., et al. (2016a). Lysine acetylation regulates the activity of Escherichia coli S-adenosylmethionine synthase. Acta Biochim. Biophys. Sin. (Shanghai) 48, 723-731. doi: 10.1093/abbs/ gmw066

Suzuki, S., Kondo, N., Yoshida, M., Nishiyama, M., and Kosono, S. (2019). Dynamic changes in lysine acetylation and succinylation of the elongation factor Tu in Bacillus subtilis. Microbiology 165, 65-77. doi: 10.1099/mic.0. 000737

Szućko, I. (2016). Sirtuins: not only animal proteins. Acta Physiol. Plant. 38:237. doi: 10.1007/s11738-016-2255-y

Tan, Y., Xu, Z., Tao, J., Ni, J., Zhao, W., Lu, J., et al. (2015). A SIRT4-like auto ADPribosyltransferase is essential for the environmental growth of Mycobacterium smegmatis. Acta Biochim. Biophys. Sin. (Shanghai) 48, 145-152. doi: 10.1093/ abbs/gmv121

Thao, S., Chen, C.-S., Zhu, H., and Escalante-Semerena, J. C. (2010). Ne-lysine acetylation of a bacterial transcription factor inhibits its DNA-binding activity. PLoS One 5:e15123. doi: 10.1371/journal.pone.0015123

Torres-Barredo, G. A., Atarashi, H., Kajikawa, A., Hirata, A., Endo, A., and Nakagawa, J. (2018). Intracellular localization of sirtuin and cell length analysis of Lactobacillus paracasei suggest possible role of sirtuin in cell division and cell shape regulation. Biosci. Biotechnol. Biochem. 82, 916-925. doi: 10.1080/ 09168451.2018.1443790

Tsang, A. W., and Escalante-Semerena, J. C. (1996). cobB function is required for catabolism of propionate in Salmonella typhimurium LT2: evidence for existence of a substitute function for CobB within the 1,2-propanediol utilization (pdu) operon. J. Bacteriol. 178, 7016-7019. doi: 10.1128/jb.178.23. 7016-7019.1996
Tsang, A. W., and Escalante-semerena, J. C. (1998). CobB, a new member of the SIR2 family of eucaryotic regulatory proteins, is required to compensate for the lack of nicotinate mononucleotide: 5, 6-Dimethylbenzimidazole phosphoribosyltransferase activity in cobT mutants during cobalamin biosynthesis i. J. Biol. Chem. 273, 31788-31794. doi: 10.1074/jbc.273.48.31788

Tucker, A. C., and Escalante-Semerena, J. C. (2010). Biologically active isoforms of CobB sirtuin deacetylase in Salmonella enterica and Erwinia amylovora. J. Bacteriol. 192, 6200-6208. doi: 10.1128/JB.00874-810

Tucker, A. C., and Escalante-Semerena, J. C. (2013). Acetoacetyl-CoA synthetase activity is controlled by a protein acetyltransferase with unique domain organization in Streptomyces lividans. Mol. Microbiol. 49, 1841-1850. doi: 10. 1016/j.jacc.2007.01.076

Umehara, T., Kosono, S., Söll, D., and Tamura, K. (2018). Lysine acetylation regulates Alanyl-tRNA synthetase activity in Escherichia coli. Genes (Basel) 9:473. doi: 10.3390/genes9100473

Van Meter, M., Mao, Z., Gorbunova, V., and Seluanov, A. (2011). Repairing split ends: SIRT6, mono-ADP ribosylation and DNA repair. Aging (Albany. NY) 3, 829-835. doi: 10.18632/aging.100389

VanDrisse, C. M., and Escalante-Semerena, J. C. (2018). In Streptomyces lividans, acetyl-CoA synthetase activity is controlled by $\mathrm{O}$-serine and $\mathrm{N} \varepsilon$-lysine acetylation. Mol. Microbiol. 107, 577-594. doi: 10.1111/mmi.13901

VanDrisse, C. M., Parks, A. R., and Escalante-Semerena, J. C. (2017). A toxin involved in Salmonella persistence regulates its activity by acetylating its cognate antitoxin, a modification reversed by CobB sirtuin deacetylase. mBio 8:e00708-17. doi: 10.1128/mBio.00708-717

Vassilopoulos, A., Fritz, K. S., Petersen, D. R., and Gius, D. (2011). The human sirtuin family: evolutionary divergences and functions. Hum. Genomics 5, 485-496. doi: 10.1186/1479-7364-5-5-485

Venkat, S., Chen, H., McGuire, P., Stahman, A., Gan, Q., and Fan, C. (2019). Characterizing lysine acetylation of Escherichia coli type II citrate synthase. FEBS J. 286, 2799-2808. doi: 10.1111/febs.14845

Venkat, S., Chen, H., Stahman, A., Hudson, D., McGuire, P., Gan, Q., et al. (2018). Characterizing lysine acetylation of isocitrate dehydrogenase in Escherichia coli. J. Mol. Biol. 430, 1901-1911. doi: 10.1016/j.jmb.2018.04.031

Vergnolle, O., Xu, H., and Blanchard, J. S. (2013). Mechanism and regulation of mycobactin fatty acyl-AMP ligase FadD33. J. Biol. Chem. 288, 28116-28125. doi: 10.1074/jbc.M113.495549

Wagner, G. R., and Payne, R. M. (2013). Widespread and enzyme-independent NEacetylation and $\mathrm{N} \varepsilon$-succinylation of proteins in the chemical conditions of the mitochondrial matrix. J. Biol. Chem. 288, 29036-29045. doi: 10.1074/jbc.M113. 486753

Walter, B. M., Morcinek-Orłowska, J., Szulc, A., Lovering, A. L., Banzhaf, M., and Glinkowska, M. (2020). Global query on bacterial sirtuin CobB interactants reveals crosstalk with PRPP synthase Prs. bioRxiv [preprint] doi: 10.1101/2020. 12.09.417477

Wang, G., Wang, Y., Zhang, L., Cai, Q., Lin, Y., Lin, L., et al. (2020). Proteomics analysis reveals the effect of Aeromonas hydrophila sirtuin CobB on biological functions. J. Proteomics 225:103848. doi: 10.1016/j.jprot.2020.10 3848

Wang, Q., Zhang, Y., Yang, C., Xiong, H., Lin, Y., Yao, J., et al. (2010). Acetylation of metabolic enzymes coordinates carbon source utilization and metabolic flux. Science 327, 1004-1007. doi: 10.1126/science.1179687

Wei, W., Liu, T., Li, X., Wang, R., Zhao, W., Zhao, G., et al. (2017). Lysine acetylation regulates the function of the global anaerobic transcription factor FnrL in Rhodobacter sphaeroides. Mol. Microbiol. 104, 278-293. doi: 10.1111/ mmi.13627

Weinert, B. T., Iesmantavicius, V., Wagner, S. A., Schölz, C., Gummesson, B., Beli, P., et al. (2013a). Acetyl-Phosphate is a critical determinant of lysine acetylation in E. coli. Mol. Cell 51, 265-272. doi: 10.1016/j.molcel.2013. 06.003

Weinert, B. T., Schölz, C., Wagner, S. A., Iesmantavicius, V., Su, D., Daniel, J. A., et al. (2013b). Lysine succinylation is a frequently occurring modification in prokaryotes and eukaryotes and extensively overlaps with acetylation. Cell Rep. 4, 842-851. doi: 10.1016/j.celrep.2013.07.024

Weinert, B. T., Moustafa, T., Iesmantavicius, V., Zechner, R., and Choudhary, C. (2015). Analysis of acetylation stoichiometry suggests that SIRT 3 repairs nonenzymatic acetylation lesions. EMBO J. 34, 2620-2632. doi: 10.15252/embj. 201591271 
Weinert, B. T., Satpathy, S., Hansen, B. K., Lyon, D., Jensen, L. J., and Choudhary, C. (2017). Accurate quantification of site-specific acetylation stoichiometry reveals the impact of Sirtuin deacetylase CobB on the E. coli acetylome. Mol. Cell. Proteomics 16, 759-769. doi: 10.1074/mcp.M117.067587

Williams, B. S., Isokpehi, R. D., Mbah, A. N., Hollman, A. L., Bernard, C. O., Simmons, S. S., et al. (2012). Functional annotation analytics of Bacillus genomes reveals stress responsive acetate utilization and sulfate uptake in the biotechnologically relevant Bacillus megaterium. Bioinform. Biol. Insights 6, 275-286. doi: 10.4137/BBI.S7977

Wua, X., Vellaichamy, A., Wang, D., Zamdborgb, L., Kelleher, L. N., Hubera, S., et al. (2013). Differential lysine acetylation profiles of Erwinia amylovora strains revealed by proteomics. Physiol. Behav. 176, 139-148. doi: 10.1016/j.jprot.2012. 12.001

Xie, L., Wang, X., Zeng, J., Zhou, M., Duan, X., Li, Q., et al. (2015). Proteome-wide lysine acetylation profiling of the human pathogen Mycobacterium tuberculosis. Int. J. Biochem. Cell Biol. 59, 193-202. doi: 10.1016/j.biocel.2014.11.010

$\mathrm{Xu}, \mathrm{H}$., Hegde, S. S., and Blanchard, J. S. (2011). The reversible acetylation and inactivation of Mycobacterium tuberculosis acetyl-CoA synthetase is dependent on cAMP. Biochemistry 50, 5883-5892. doi: 10.1021/bi200156

Xu, Z., Zhang, H., Zhang, X., Jiang, H., Liu, C., Wu, F., et al. (2019). Interplay between the bacterial protein deacetylase CobB and the second messenger c-diGMP. EMBO J. 38:e100948. doi: 10.15252/embj.2018100948

Yadav, G. S., Ravala, S. K., Malhotra, N., and Chakraborti, P. K. (2016). Phosphorylation modulates catalytic activity of mycobacterial sirtuins. Front. Microbiol. 7:677. doi: 10.3389/fmicb.2016.00677

You, D., Wang, M. M., and Ye, B. C. (2017). Acetyl-CoA synthetases of Saccharopolyspora erythraea are regulated by the nitrogen response regulator GlnR at both transcriptional and post-translational levels. Mol. Microbiol. 103, 845-859. doi: 10.1111/mmi.13595

You, D., Yao, L. L., Huang, D., Escalante-Semerena, J. C., and Ye, B. C. (2014). Acetyl coenzyme a synthetase is acetylated on multiple lysine residues by a protein acetyltransferase with a single Gcn5-type N-acetyltransferase (GNAT) domain in Saccharopolyspora erythraea. J. Bacteriol. 196, 3169-3178. doi: 10. 1128/JB.01961-1914

You, D., Yin, B. C., Li, Z. H., Zhou, Y., Yu, W. B., Zuo, P., et al. (2016). Sirtuindependent reversible lysine acetylation of glutamine synthetases reveals an autofeedback loop in nitrogen metabolism. Proc. Natl. Acad. Sci. U S A. 113, 6653-6658. doi: 10.1073/pnas.1525654113

Yu, B. J., Kim, J. A., Moon, J. H., Ryu, S. E., and Pan, J. G. (2008). The diversity of lysine-acetylated proteins in Escherichia coli. J. Microbiol. Biotechnol. 18, 1529-1536.

Yu, S., Ren, J., Ni, J., Tao, J., Lu, J., and Yao, Y. F. (2016). Protein acetylation is involved in Salmonella enterica serovar typhimurium virulence. J. Infect. Dis. 213, 1836-1845. doi: 10.1093/infdis/jiw028
Yuan, H., and Marmorstein, R. (2012). Structural basis for Sirtuin activity and inhibition. J. Biol. Chem. 287, 42428-42435. doi: 10.1074/jbc.R112. 372300

Zhang, J., Sprung, R., Pei, J., Tan, X., Kim, S., Zhu, H., et al. (2009). Lysine acetylation is a highly abundant and evolutionarily conserved modification in Escherichia coli. Mol. Cell. Proteomics 8, 215-225. doi: 10.1074/mcp.M800187MCP200

Zhang, Q., Zhou, A., Li, S., Ni, J., Tao, J., Lu, J., et al. (2016). Reversible lysine acetylation is involved in DNA replication initiation by regulating activities of initiator DnaA in Escherichia coli. Sci. Rep. 6:30837. doi: 10.1038/srep30837

Zhang, Q.-F., Zhang, Q., Gu, J., Gong, P., Wang, X.-D., Wang, X., et al. (2013). Reversibly acetylated lysine residues play important roles in the enzymatic activity of Escherichia coli N-hydroxyarylamine O-acetyltransferase. FEBS J. 280, 1966-1979. doi: 10.1111/febs.12216

Zhang, X., Ameer, F. S., Azhar, G., and Wei, J. Y. (2021). Alternative splicing increases sirtuin gene family diversity and modulates their subcellular localization and function. Int. J. Mol. Sci. 22:473. doi: 10.3390/ijms220 20473

Zhao, K., Chai, X., and Marmorstein, R. (2004). Structure and substrate binding properties of CobB, a Sir2 homolog protein deacetylase from Escherichia coli. J. Mol. Biol. 337, 731-741. doi: 10.1016/j.jmb.2004.01.060

Zhou, Y., Li, Z. H., and Ye, B. C. (2018). SacAcuA/SacSrtN system modulates the metabolism by controlling the special proteins in Saccharopolyspora erythraea. Ann. Microbiol. 68, 135-143. doi: 10.1007/s13213-018-13251323

Conflict of Interest: The authors declare that the research was conducted in the absence of any commercial or financial relationships that could be construed as a potential conflict of interest.

Publisher's Note: All claims expressed in this article are solely those of the authors and do not necessarily represent those of their affiliated organizations, or those of the publisher, the editors and the reviewers. Any product that may be evaluated in this article, or claim that may be made by its manufacturer, is not guaranteed or endorsed by the publisher.

Copyright (c) 2021 Gallego-Jara, Ortega, Lozano Terol, Sola Martínez, Cánovas Díaz and de Diego Puente. This is an open-access article distributed under the terms of the Creative Commons Attribution License (CC BY). The use, distribution or reproduction in other forums is permitted, provided the original author(s) and the copyright owner(s) are credited and that the original publication in this journal is cited, in accordance with accepted academic practice. No use, distribution or reproduction is permitted which does not comply with these terms. 